\title{
Monitoring Tensile Fatigue of Superelastic NiTi Wire in Liquids by Electrochemical Potential
}

\author{
Jan Racek $^{1} \cdot$ Marc Stora $^{1,2} \cdot$ Petr Šttner $^{1} \cdot$ Luděk Heller $^{1} \cdot$ Jaromir Kopeček $^{1} \cdot$ \\ Martin Petrenec ${ }^{3}$
}

Published online: 24 June 2015

(C) ASM International 2015

\begin{abstract}
Fatigue of superelastic NiTi wires was investigated by cyclic tension in simulated biofluid. The state of the surface of the fatigued $\mathrm{NiTi}$ wire was monitored by following the evolution of the electrochemical open circuit potential (OCP) together with macroscopic stresses and strains. The ceramic $\mathrm{TiO}_{2}$ oxide layer on the NiTi wire surface cannot withstand the large transformation strain and fractures in the first cycle. Based on the analysis of the results of in situ OCP experiments and SEM observation of cracks, it is claimed that the cycled wire surface develops mechanochemical reactions at the NiTi/liquid interface leading to cumulative generation of hydrogen, uptake of the hydrogen by the NiTi matrix, local loss of the matrix strength, crack transfer into the NiTi matrix, accelerated crack growth, and ultimately to the brittle fracture of the wire. Fatigue degradation is thus claimed to originate from the mechanochemical processes occurring at the excessively deforming surface not from the accumulation of defects due to energy dissipative bulk deformation processes. Ironically, combination of the two exciting
\end{abstract}

Electronic supplementary material The online version of this article (doi:10.1007/s40830-015-0020-5) contains supplementary material, which is available to authorized users.

Jan Racek

racek@fzu.cz

Petr Šittner

sittner@fzu.cz

1 Institute of Physics ASCR, Na Slovance 2, 18221 Prague, Czech Republic

2 French Institute for Advanced Mechanics (IFMA), campus de Clermont-Ferrand, 27 rue Roche Genes, 63170 Aubiere, France

3 TESCAN, Brno, Czech Republic properties of $\mathrm{NiTi}$-superelasticity due to martensitic transformation and biocompatibility due to the protective $\mathrm{TiO}_{2}$ surface oxide layer-leads to excessive fatigue damage during cyclic mechanical loading in biofluids.

Keywords Nitinol - Shape memory alloy - Corrosion . Fatigue $\cdot$ Tensile test $\cdot$ Electrochemistry $\cdot$ Hydrogen embrittlement

\section{Introduction}

NiTi-based alloys have established themselves as key shape memory alloy material for wide range of engineering applications [1] exploiting their unique functional thermomechanical behaviors due to the martensitic transformation driven by the stress and temperature. The key phenomena are the large recoverable superelastic and shape memory strains and strong stress-temperature coupling. When trying to utilize these phenomena in applications designs, NiTi alloys are considered to be exposed to long-time cyclic variations of large stress ( $<700 \mathrm{MPa})$, large reversible strains $(<7 \%)$, wide range of temperatures $\left(-150{ }^{\circ} \mathrm{C}\right.$ to $\left.+120{ }^{\circ} \mathrm{C}\right)$, or even corrosive environments (water, blood, biofluids, oil, vapors, hydrogen). Depending on the imposed strain amplitude, the $\mathrm{NiTi}$ alloys exhibit thousands to millions of cycles to failure in (thermo)mechanical cycling, no other metallic materials can even approach such a superior fatigue performance at comparable conditions. In successful practical applications, however, the conditions are frequently limited. In this context, the "limited thermomechanical fatigue performance of NiTi" represents an obstacle hindering further development of many engineering applications of $\mathrm{NiTi}$ (superelastic stents, orthodontic implants and tools, vibration damping elements, actuator springs, HTSMA actuators). 
Fatigue of phase-transforming NiTi has been investigated in the literature both experimentally [2-8] and theoretically [7, 8]. Although the stress and/or temperature driven martensitic transformation proceeds cyclically in all fatigue tests, fatigue of superelastic medical stents, orthodontic wires and implants, thermomechanical fatigue of actuator wires or springs or fatigue of high temperature actuators seems to be governed by different rules and hence must be dealt with separately.

Functional fatigue (cyclic degradation of functional response) [3] and structural fatigue [6] have to be distinguished and possible links between them shall be identified. In spite of the attention paid to the drift of the cyclic stressstrain-temperature responses in the literature [3, 4], it is not still clear how the drift is exactly related to the structural fatigue. There seems to be a general agreement in the literature on a dissipation energy-based fatigue criterion [8] - the more dissipated energy is stored in the material during the transformation cycle, the shorter is the fatigue lifetime. In other words, if there is no energy dissipation, there should be no fatigue and full strain superelastic cycling shall be feasible for millions of cycles. But energy dissipation and hysteresis are intrinsically related to the martensitic transformation and lattice defects are created by phase interfaces propagating during cyclic martensitic transformations [9]. The accumulating defects lead to the microstructure evolution during the thermomechanical cycling [10] which is believed to control the fatigue performance $[3,6]$. But is this really responsible for the experimentally observed preliminary failure of superelastically cycled NiTi? No one proved that yet. Reliable experimental data on the link from the microstructure evolution to the structural fatigue performance of NiTi are still missing. Many researchers, particularly in the community of researchers from NiTi suppliers and medical device companies, believe that the size and shape of nonmetallic inclusions, which cannot deform together with the superelastic NiTi, are primarily responsible for its poor fatigue performance. There are many problems related with the experimental investigation superelastic fatigue of NiTi wire in tension. If the tests are performed at slow strain rates, they are painfully long. At higher strain rates in the air, however, the stress-strain responses are affected by the heat effects [11], particularly to the latent heat, thermomechanical coupling and heat exchange with the environment. If the fast cyclic loading tests are performed on NiTi wires immersed in fluids, this is not so serious due to much better heat exchange with the fluid, but there is a danger of environmental effects affecting the fatigue performance. The optimum solution for fatigue testing of NiTi wires seems to be mechanical cycling at high frequencies in fluids. Rotating bending fatigue testing on wires [2] or pulsating fatigue on NiTi stents [12] is popular in the medical device field. A complication is that the acting surface stresses and strains are not directly measured in rotating bending fatigue tests and interpretation of the results is less straightforward compared to the case of a wire fatigued in cyclic tension.

This work deals with an experimental investigation of structural fatigue of superelastic NiTi wire transforming cyclically in simulated biofluids at constant body temperature under cyclic tension. A motivation comes from our earlier work on fatigue of superelastic NiTi springs cycled in simulated biofluids [13-15] targeting particularly the problem of unexpected failure of gastrointestinal esophageal, and/or tracheal NiTi stents [16] braided from NiTi wires or orthodontic wires which are exposed to mechanical loads in corrosive biofluids-i.e., NiTi implants which transform martensitically in the body. Clinical reports of unexpected random failures of implanted braided NiTi stents show that the NiTi wires of implanted stents unexpectedly fractured soon after implantation and the explanted stents exhibited serious embrittlement [17]. Physical origin of the failure remained unclear and corrosion was simply blamed responsible without specifying the exact fatigue damage and failure mechanism.

In a sharp contrast with that, there is vast experimental evidence in the biomedical literature [18-20] suggesting that carefully engineered $\mathrm{TiO}_{2}$ surface oxide layer on the superelastic NiTi wire separating the liquid environment from the bare metal provides the NiTi with safe protection even against highly corrosive bioenvironments. The $\mathrm{TiO}_{2}$ layer created by furnace oxidation on NiTi consists presumably of mixed polycrystalline anatase with amorphous phase [21]. XPS measurements show excess Ni on top of $\mathrm{TiO}_{2}$ surface (native or heat-treated oxide) in a form of $\mathrm{Ni}$ "hut-clusters" ( $\sim 5 \mathrm{~nm})$ top [22, 23]. The experimentally observed growth of metallic and ionic bonded $\mathrm{Ni}-\mathrm{O}$ overlayer on the top of $\mathrm{TiO}_{2}$ was explained using the application of the valence bond theory [22, 24].

A question whether the unexpected embrittlement and fracture of implanted NiTi stents shall be ascribed to mechanical fatigue stemming from the bulk or to corrosion attack stemming from the surface was addressed in our recent work [13-15]. It was concluded that both are probably involved in the gradual fatigue degradation and that cracking of the thin $\mathrm{TiO}_{2}$ oxide layer (grown by thermal oxidation on the wire surface during the shape setting heat treatment) probably plays a key role in the degradation process. The hard ceramic oxide cannot withstand large surface strains due to stress-induced martensitic transformation and fractures when the surface strains exceed $1 \%$ [15]. If the surface oxide does not fracture, the number of cycles to failure quickly increases with the decreasing strain amplitude and reaches a run out number of cycles. It was concluded that the fatigue limit evaluated in NiTi spring tests $[13,15]$ is controlled by the surface oxide damage. The actual failure mechanism, however, remained unclear. 
Since the NiTi stent is typically heavily deformed during the microinvasive implantation, the oxide fractures and the wire of the implanted NiTi stent is protected against corrosion by the "cracked $\mathrm{TiO}_{2}$ oxide." Figure 1 shows a SEM image of the cracked oxide layer on a superelastic NiTi wire transforming via shear band propagation in tension [14]. It can be seen that the oxide layer behind the shear band front is broken by high density of regularly spaced thin oxide cracks oriented perpendicularly to the wire axis. Let us point out that, since the oxide cracks are tiny and close upon unloading, they can only be observed by SEM on the stretched wire surface (Fig. 1).

The presence of the oxide cracks, however, does automatically imply neither degradation of the corrosion properties nor degradation of the fatigue performance of NiTi, though the cracking has been found to result in accelerated $\mathrm{Ni}$ release [23]. The oxide layer is too thin to pose any mechanical threat to the wire. Moreover, the damage to the oxide layer caused by the excessive surface deformation gets quickly "repaired" by fast passivation reaction in the biofluid [25]. Nevertheless, NiTi springs superelastically cycled in water and/or biofluids survive only several thousands of tensile cycles (much less than in the air [13] for the same stroke amplitude) and ultimately fail in a brittle manner. This clearly suggests that the liquid environment does have an adverse effect on the fatigue performance of superelastically cycled

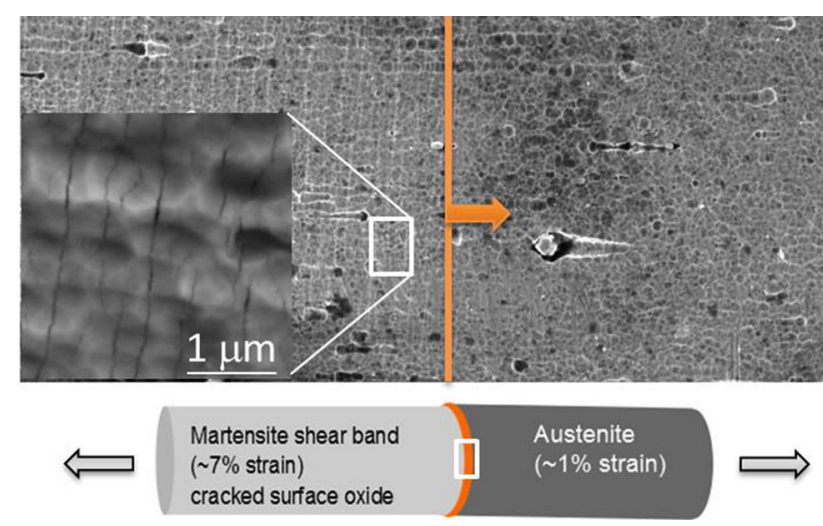

Fig. 1 Superelastic NiTi wire deforms in tension via propagation of shear band front(s), separating the newly formed martensite shear band ( $\sim 7 \%$ strain) from the rest of the still austenitic wire $(\sim 1 \%$ strain). The shear band front is seen as a circle along the circumference of the wire, where its diameter slightly decreases. Within the wire, shear band front has a shape of a cone pointing towards the martensite [51]. The internal stress is locally elevated on the surface of this cone compared to the rest of the wire [51]. The stress-induced martensitic transformation takes place mainly within this shear band front as it travels along the wire. The $\mathrm{TiO}_{2}$ surface oxide wire cannot deform homogeneously to $7 \%$ and fractures upon straining. The moving shear band front thus leaves behind a "cracked surface oxide" containing regularly spaced, thin oxide cracks oriented perpendicularly to the wire axis. As the oxide cracks are extremely thin, they can be observed by SEM only under applied stress since they close as the shear band moves back upon unloading
NiTi springs. It brought us to the suspicion that chemical reactions proceeding periodically within the cracked surface oxide layer might be responsible for the poor fatigue performance of NiTi wire immersed in biofluid.

Chemical processes at the solid/liquid interface of deforming metals can be conveniently studied by electrochemical methods applied during mechanical loading [2629]. In such in situ tests, the deformed metal serves as the working electrode connected in electrochemical cell with inert reference electrode and processes on its surface are tracked down by evaluating electrochemical signals (potential, current, impedance, etc.) during the mechanical loads. These methods were applied in the literature to plastically deformed metals [29]. Mechanochemistry of surface reactions on deformed metals has been explored theoretically [30]. First experiments on NiTi for simultaneous assessment of the electrochemical and structural fatigue properties were designed by Neelakantan et al. [27]

We have recently performed in situ electrochemical experiments on NiTi wires and springs superelastically cycled in fluids [31]. Basic experiment involves evaluation of the electrochemical Open Circuit Potential/OCP/(potential difference of the working electrode against the reference electrode) during the cyclic tensile loads. The experimentally measured OCP generally depends on the metal, electrolyte, surface layer, temperature, and time of the exposure of the material to the electrolyte. If all this is constant, the experimentally measured changes of the OCP reflect the mechanically induced damage of the surface oxide and the progress of the chemical reactions on the surface triggered by this damage. Potentially very interesting information on the mechanochemical processes occurring on the surface of the fatigued wire can be obtained in this way. In particular, the in-situ recorded OCP signal reflects periodic straining of the surface oxide, cracking of the oxide at large strain amplitudes, crack opening/closing upon tensile cycling or chemical passivation of the mechanically damaged surface.

Physical meaning of the results, particularly how the in situ recorded OCP signals are related to the mechanical data and fatigue performance of $\mathrm{NiTi}$, however, is not clear yet since the method has never been systematically applied to NiTi. This is the main goal of this work.

\section{Experimental Methods}

\section{Superelastic NiTi Wires}

Medical grade superelastic NiTi wires were studied in present experiments (Fort Wayne Metals \#1, $d=0.2 \mathrm{~mm}$, straight wires with etched, mechanically polished and naturally oxidized surface). The wire samples were prepared from the same batch, given heat treatment in a fluid 
furnace at $510{ }^{\circ} \mathrm{C}$ for $4 \mathrm{~min}$ and cooled in water for $3 \mathrm{~min}$. As a result of this treatment, all wire samples used in this study have the same microstructure and same surface oxide as the NiTi spring samples studied in our earlier closely related work [13-15] $\left(\sim 70 \mathrm{~nm} \mathrm{TiO}_{2}\right.$ layer with excess $\mathrm{Ni}$ on the top and $\sim 40$-nm-thick Ni-enriched layer underneath [14]). Capillaries were clamped on both ends of the wire sample, electrically isolated with Teflon and epoxy and gripped into the testing rig. All wire samples were $27 \mathrm{~mm}$ long and had equal surface area exposed to the electrolyte as the working electrode. All tests were performed on virgin wires.

\section{Electrochemical Testing of Superelastic NiTi Wire Deformed in Liquids}

The electrochemical measurements of open circuit potential (OCP), which is also called the corrosion potential $\left(E_{\text {corr }}\right)$, were performed in a $0.9 \% \mathrm{NaCl}$ solution at temperature $37^{\circ} \mathrm{C}\left(25,40,50{ }^{\circ} \mathrm{C}\right)$ using a GAMRY Reference 600 potentiostat. The acidity of the solution was adjusted to $\mathrm{pH}=3$ by adding few drops of dilute $\mathrm{HCl}$ in order to simulate physiological and corrosive environment the medical stents are exposed to.

The volume of the solution in the electrochemical cell was $130 \mathrm{ml}$. Potentials were measured against a reference silver chloride electrode $(\mathrm{Ag} / \mathrm{AgCl},-0.025 \mathrm{~V}$ vs $\mathrm{SCE})$. The surface area of the thin NiTi wire acting as the working electrode was as small as $0.170 \mathrm{~cm}^{2}$. This lead to very low electrochemical current, suppression of capacitive currents and ensured non-polarizability of the reference electrode. The distance between the reference and working electrode was less than $1 \mathrm{~mm}$. There was no need to use IR compensation.

When a metal such as nickel or titanium is corroding in an acid solution, both the anodic (Eq. 1), and cathodic (Eq. 2) reactions occur simultaneously on the metal surface.

$\mathrm{M}=\mathrm{M}^{+}+\mathrm{e}^{-}$
$\mathrm{H}^{+}+\mathrm{e}^{-}=\mathrm{H}$

The reactions gradually change potential to a compromised value preventing motion of ions across the metal/ liquid interface. The potential of the working electrode measured with respect to the reference electrode is the OCP. Thermodynamic driving force of electrochemical potential for each half-cell reaction (1) and (2) is given by Nernst Eq. 3.

$E_{\text {cell }}=E_{\text {cell }}^{0}-\frac{R T}{z F} \ln Q$

$E_{\text {cell }}[V]$ is the cell potential, $E_{\text {cell }}^{0}[V]$ is the standard cell potential, $R\left[\mathrm{Jmol}^{-1} \mathrm{~K}^{-1}\right]$ is a gas constant, $T[K]$ is the absolute temperature, $z$ is the number of moles of electrons transferred in the half-cell reaction, $F$ is the Faraday constant, $Q$ is a reaction quotient, where $Q=\frac{C_{\text {red }}}{C_{\text {ox }}}$, with $c_{\text {red }}$ being the concentration of the reduced form, and $c_{\mathrm{ox}}$ is the concentration of the oxidized form.

The OCP value measured in the in situ electrochemical test on NiTi wire acting as the working electrode in the electrochemical cell thus characterizes an electrochemical quasiequilibrium at the NiTi/liquid interface reflecting the kinetics of both half-cell reactions. When this equilibrium is changed by the mechanical deformation of the NiTi surface, the kinetics of chemical reactions changes and the experimentally measured OCP reflects this change and evolves in time. Increase of the OCP in time evidences progress of the oxidation process (Eq. 1), while decrease suggests prevailing reduction process (Eq. 2) at the NiTi/ liquid interface.

\section{In Situ Electrochemical Testing During Tensile Cycling of Superelastic NiTi Wire}

Dedicated equipment for in situ electrochemical testing of metallic wires cyclically deformed in liquids was designed and constructed (Fig. 2). The scheme of the experimental setup used for in situ evaluation of the OCP of the superelastic NiTi wires subjected to tensile cycling held at constant temperature is shown on the top of Fig. 2. The equipment consists of a thermally controlled sealed basin with electrolyte, linear stepping motor, load cell, laser extensometer, electrically isolated grips, wire sample as the working electrode, reference electrode, potentiostat, control program and data acquisition system in LabView. To prevent leakage of the electrolyte through the holes for the moving wire, low pressure is kept in the sealed electrochemical cell. Reference electrode is on the top of the box.

Two types of in situ electrochemical tests were performed: (a) kinetics experiments on deformed NiTi wires and (b) cyclic experiments. In the kinetics experiments, the wires were strained (unloaded) in the liquid up to a preset strain level using defined strain rate, while the OCP response was evaluated during the straining and predefined period of time afterwards. In the cyclic experiments, the wires were subjected to cyclic tensile tests characterized by test parameters as e.g., mean strain, strain amplitude, strain rate, temperature and the OCP response was continuously evaluated during the cycling.

As introduced above, the experimentally recorded OCP signal reflects mechanically induced local breakdown of the passive oxide layers triggering the chemical reactions on the wire surface. Note that the oxide damage is brought about by the excessive superelastic deformation caused by the stress-induced MT in the NiTi matrix underneath the 

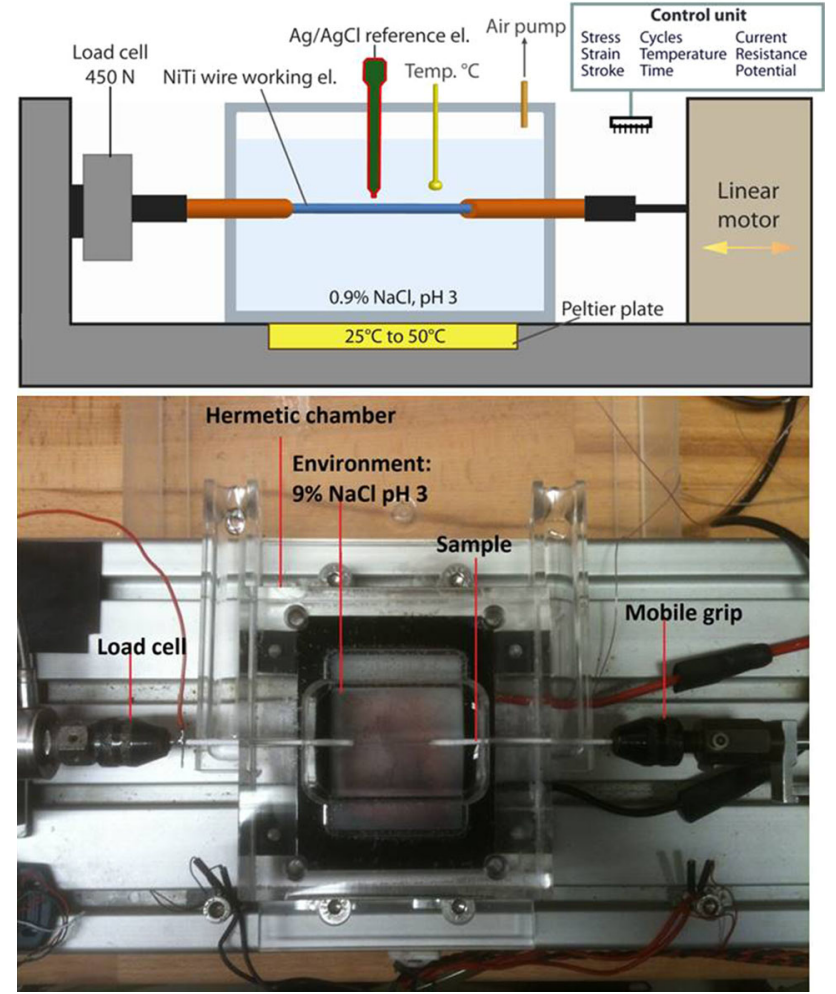

Fig. 2 Experimental setup for in situ electrochemical testing during cyclic tensile deformation of metallic wires in fluids. Scheme of the equipment (top) and photo of the rig with mounted NiTi wire sample (bottom). Force, elongation, temperature, and electrochemical potential OCP are continuously recorded during the cyclic tensile test

oxide. While the damage to the oxide layer caused by the $\mathrm{NiTi}$ deformation is instantaneous, the progress of chemical reaction (Eqs. 1,2) is delayed. The surface strains vary periodically during the cyclic tensile tests, unfortunately the localized deformation of NiTi in tension complicates the issue significantly.

The in situ recorded OCP signal was stored together with external force, elongation and temperature. The OCP sampling rate in kinetics tests was set at $1000 \mathrm{~Hz}$. High sampling rate is essential to evaluate the minimum value of OCP established as a result of deformation-induced damage followed by very fast passivation of NiTi wire. The OCP minimum approaches the true corrosion potential of the bare NiTi alloy exposed to electrolyte.

\section{Characterization of the Cracked Surface Oxide by SEM}

SEM/FIB techniques were employed to characterize the surface defects introduced by the cyclic tensile deformation. SEM observation was performed using FIB/SEM TESCAN FERA3 electron microscope on the NiTi wire sample, which was previously subjected to the tensile fatigue test (strain rate $=1 \% \mathrm{~s}^{-1}$, simulated biofluid, $\left.T=37^{\circ} \mathrm{C}, N^{\mathrm{F}}=1500\right)$. The wire was stretched using a special tensile holder to $6 \%$ strain. A part of the wire was still in austenite structure with closed surface oxide cracks ( $\sim 1 \%$ strain), while the majority of the wire was in the martensite shearband $(\sim 7 \%$ strain) with surface oxide cracks open. Subsequently, cross sections were cut through the wire in areas of interest using the FIB with $\mathrm{Ga}^{+}$ions using the TESCAN LYRA SEM perpendicularly to the major oxide cracks. Final polishing was carried out using current of $560 \mathrm{pA}$ and cracks penetrating into the NiTi matrix were studied on the polished cross sections. Finally, $3 \mathrm{D}$ reconstruction of subsurface distribution of microcracks was performed using cross-sectioning method by automated FIB milling combined with SE scanning of the bulk $40 \times 10 \times 17 \mu \mathrm{m}$ (400 slices in steps of $100 \mathrm{~nm})$.

\section{Experimental Results}

\section{Cyclic Tensile Deformation of Superelastic NiTi Wire in Biofluid and in the Air}

Cyclic tensile tests were performed on NiTi wires in position control under wide range of testing conditions (strain amplitude, strain rate, temperature). Force, elongation, temperature, and electric resistance were continuously recorded. Figure 3 compares cyclic stress-strain response of NiTi wire subjected to cyclic tensile loading tests at temperature $T=37{ }^{\circ} \mathrm{C}$ using strain rate $1 \% \mathrm{~s}^{-1}$ in biofluid (Fig. 3a) and in the air (Fig. 3c). The stress-strain responses evolve quickly in the first 100 cycles and very slowly in the rest of the lifetime (Fig. 3b). Gradual evolution of transformation stress, hysteresis, and residual strain with number of cycles in the first 100 cycles suggests that the wire microstructure changes mainly in the early stages of the test. There are significant differences between the stress-strain curves measured in the liquid and in the air (higher forward transformation stress, wider hysteresis, and larger residual strain in the air). We ascribe these differences to the latent heat effects [11] at relatively high strain rate, which are supposed to be different in air and fluid and the wire hence deforms at different temperatures though nominally the temperature $T=37{ }^{\circ} \mathrm{C}$ was held constant in both cases.

The essential point is that the number of cycles till failure is significantly lower in the liquid than in the air. In the small amplitude range, the NiTi wire survived 100 mil. cycles at strain amplitude $0.5 \%$ while only $N^{\mathrm{f}}=4000$ cycles in biofluid. This suggests that the fatigue limit is different in the liquid and in the air. We did not perform sufficient number of tests at different stress amplitudes to construct Wohler curves, but we made enough tests to learn 

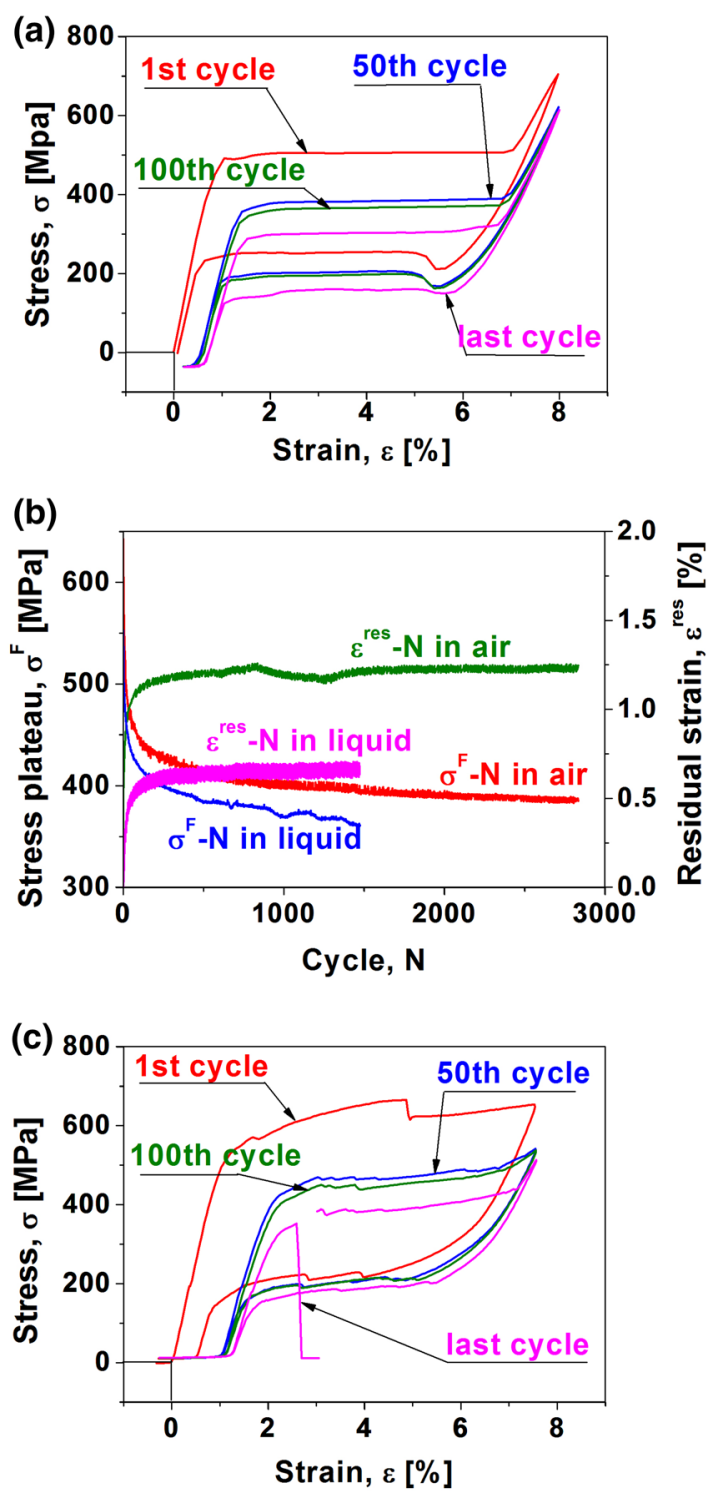

Fig. 3 Superelastic fatigue test of $\mathrm{NiTi}$ wire in tension (strain rate $=1 \% \mathrm{~s}^{-1}$, simulated biofluid, air, $T=37{ }^{\circ} \mathrm{C}$ ): a stress-strain response in 1st, 50th, 100th, and last tensile cycle in the liquid, b evolution of the residual strain, $\varepsilon^{\text {res }}$ and transformation stress, $\sigma^{\mathrm{F}}$ at $4 \%$ strain upon cycling in air and in liquid, $\mathbf{c}$ stress-strain response in 1st, 50th, 100th, and last tensile cycle in air

that the experimental results under given test conditions (strain amplitude, mean strain, strain rate, temperature, environment) are reproducible with a very small scatter $\Delta N^{\mathrm{f}}<5 \%$. This is due to the fact that all samples have same microstructure, same surface oxide and particularly that all tests are performed in thermally controlled chamber (air or liquid) isolated from the surrounding environment. The fact that number of cycles till failure is lower in the biofluid than in the air turned our attention towards the surface $\mathrm{TiO}_{2}$ oxide, as already explained in the introduction. We started to doubt whether the evolution of the bulk microstructure, which is responsible for drift of the cyclic stress-strain curve upon cycling (Fig. 3c), has a decisive impact on the fatigue lifetime of $\mathrm{NiTi}$ wires cycled in biofluid. In other words, we started to suspect that the fatigue damage originates from the mechanochemical processes at the wire surface, as common for the environmentally assisted fatigue. Hence, we decided to monitor the accumulation of the surface damage during tensile cycling by employing the in situ OCP method.

\section{OCP Kinetics Tests on Strained NiTi Wires}

Before the in situ OCP cyclic experiments, kinetics in situ OCP experiments on virgin a deformed NiTi wires were performed with the aim to obtain basic information on the kinetics of the damage and passivation processes on the surface of the immersed wire as follows. The NiTi wire was immersed into the simulated biofluid, connected into the electrochemical circuit as the working electrode against the reference electrode and evolution of the OCP in time was recorded (Fig. 4). The OCP gradually increases in time as the passivation processes take place. After $3600 \mathrm{~s}$, the wire was deformed using a defined strain rate up to a preset value of tensile strain and kept under constrained length for another $3600 \mathrm{~s}$. The OCP was continuously recorded together with force, elongation, and temperature. Finally, the wire was unloaded using the same strain rate, while $\mathrm{OCP}$, force, elongation, and temperature were recorded for another $3600 \mathrm{~s}$ again. Figure $4 \mathrm{a}$ shows, as an example, results of a test performed by deforming the wire using strain rate $1 \% \mathrm{~s}^{-1}$ up to the middle of the superelastic plateau (3\% strain). Detailed evolution of the strain, stress, and OCP recorded during the loading and unloading events is shown in Fig. 4b and c, respectively. After the wire is deformed, a part of it exists in the shear band (Fig. 1) and the rest exists in elastically loaded austenite phase. The surface of the strained wire is thus covered partly by the cracked oxide and partly by the strained oxide layer. In a response to the straining, the OCP instantaneously decreases when the martensitic transformation starts (Fig. 4b) but quickly reaches a minimum and starts to increase even before the preset strain $3 \%$ is reached. It is assumed that the OCP decreases due to the damage caused by the tensile deformation to the surface oxide (nucleation of a shear band and cracking of the surface oxide layer on top of it) and increases due to the passivation processes occurring as a response to the damage of the oxide layer. After $3600 \mathrm{~s}$, the in situ recorded OCP reaches the predeformation value (Fig. 4a). The OCP decreases also upon unloading (Fig. 4c) when reverse transformation starts but much less than during forward loading and the pre-event OCP value is restored in just few seconds.

In order to better understand the kinetics of the passivation processes, experiments were performed using 

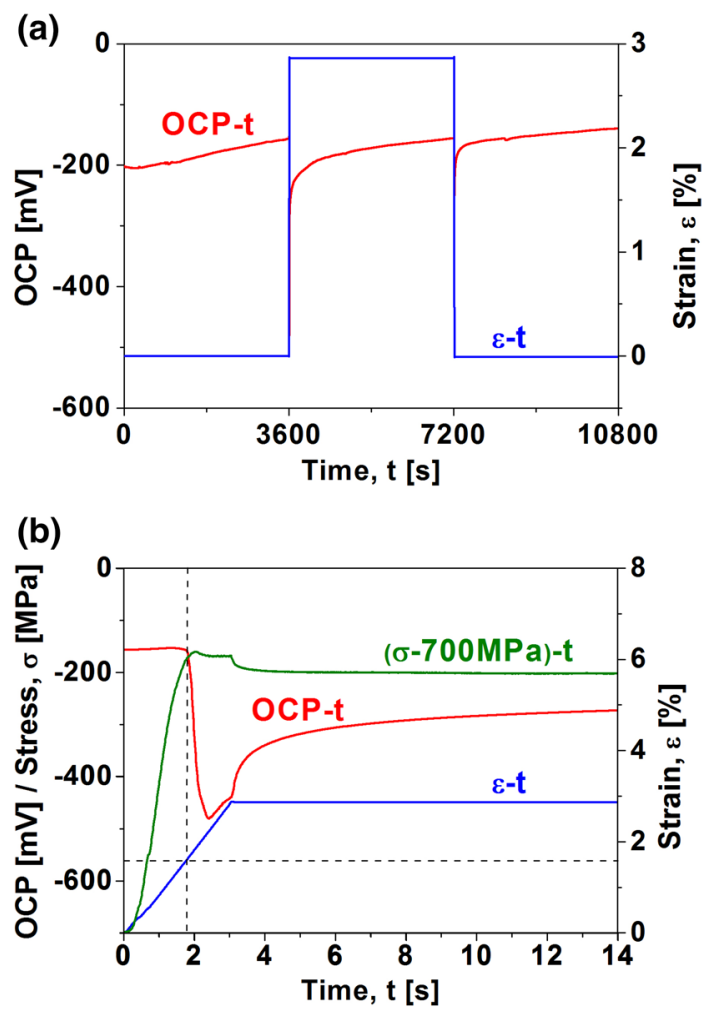

(c)

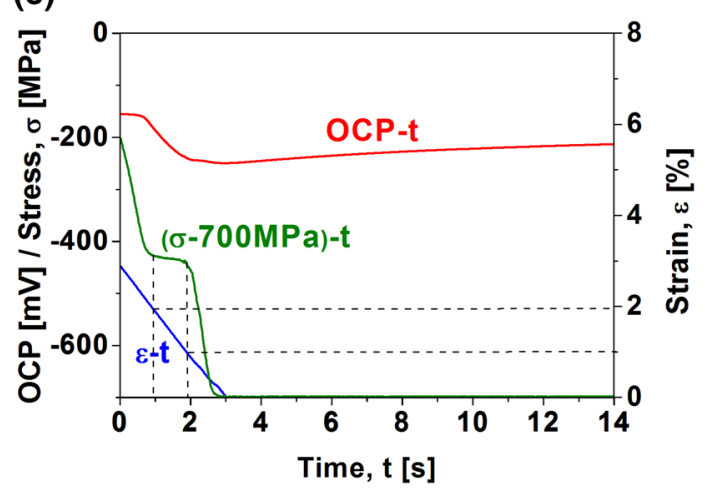

Fig. 4 OCP kinetics test. Evolution of the OCP on the NiTi wire deformed to $3 \%$ strain and unloaded (strain rate $=1 \% \mathrm{~s}^{-1}$, simulated biofluid, $T=37{ }^{\circ} \mathrm{C}$ ): a overview of the test, detail of the time evolution of the strain, stress, and OCP when the wire is deformed (b), and unloaded (c). Dashed lines show the austenite and martensite elastic strain limits

different maximum strains and two different strain rates 1 and $180 \% \mathrm{~s}^{-1}$. The results are presented in Figs. 5, 6, 7, and 8 . Figure 5 provides an overview on all kinetics experiments. One can see that there is a qualitative difference between the OCP responses recorded in kinetics tests with the maximum strain in the elastic range $(0.8 \%)$ and in tests with larger strains $(1.5-3 \%)$. The OCP decreases in response to straining already in the elastic range but, when the surface oxide fractures in tests with larger strains, there is a deep OCP minimum at the moment of the deformation. Figures 6 and 7 show the evolution of the OCP together with the evolution of stress and strain in detail. If the maximum applied strain remains within the elastic range (Fig. 6a), the OCP decrease is small and the passivation is negligible and very slow. If the applied strain is larger within the transformation range, the instant OCP decrease is large and is followed by fast passivation giving rise to the deep OCP minimum. The OCP, however, starts to fall before the martensitic transformation starts (before the shear band nucleates). The passivation is assumed to start right when the shear band nucleates and hence massively proceeds already during the straining in the slow strain rate tests (Fig. 6c, d). The OCP minimum on the kinetics curve reaches $\sim-450 \mathrm{mV}$ and seems not to depend much on the maximum strain at larger applied strains. This was somehow surprising since the area of the damaged surface evidently increases with the increasing maximum strain. It motivated us to perform similar experiments with higher strain rate $180 \% \mathrm{~s}^{-1}$ to arrive closer to the real OCP minimum. The results (Figs. 5b, 7, 8) are, however, rather similar. The OCP minimum is indeed much deeper $\sim-600 \mathrm{mV}$ (Figs. $5 \mathrm{~d}$, 7d) but it is still observed before the end of loading and still does not depend much on the maximum strain at larger strains. The pronounced stress relaxation after the end of the tensile loading (Fig. $7 \mathrm{c}, \mathrm{d}$ ) is due to the more pronounced latent heat effects at higher strain rate. Figure 8 shows that the drop of the OCP minimum is clearly related with the onset of stress-induced martensitic transformation and how it varies with the applied strain.

Regardless of the applied strain, the OCP of the deformed wire increases in the long run $(\sim 3600 \mathrm{~s})$ up to starting level in all kinetics tests, similarly as shown in Fig. 4a. It shall be noted that, except the lowest applied strains, the NiTi wire is exposed to the liquid in partially martensitic state with the surface oxide cracks opened. The surface oxide is yet virgin (uncracked) in the rest of the wire, though homogeneously strained to about $1 \%$ due to the external stress applied in the kinetics test. Please note that, as a consequence of the strain localization in shear bands, the oxide cracks on top of the shear band are kept in opened state by the surface strain $\sim 7 \%$, regardless of the actual magnitude of the applied strain.

As the experimentally measured evolution of the OCP is an integral value from the whole wire, modeling has to be employed to interpret the meaning of the in situ OCP experimental data in view of the actual processes occurring at the wire surface upon straining. This exceeds the scope of this work. Here, the aim was mainly to overview what kind of in situ OCP information can be obtained from the kinetics tests.

It was found that, by following the evolution of the OCP of the wire subjected to straining, we can detect the 

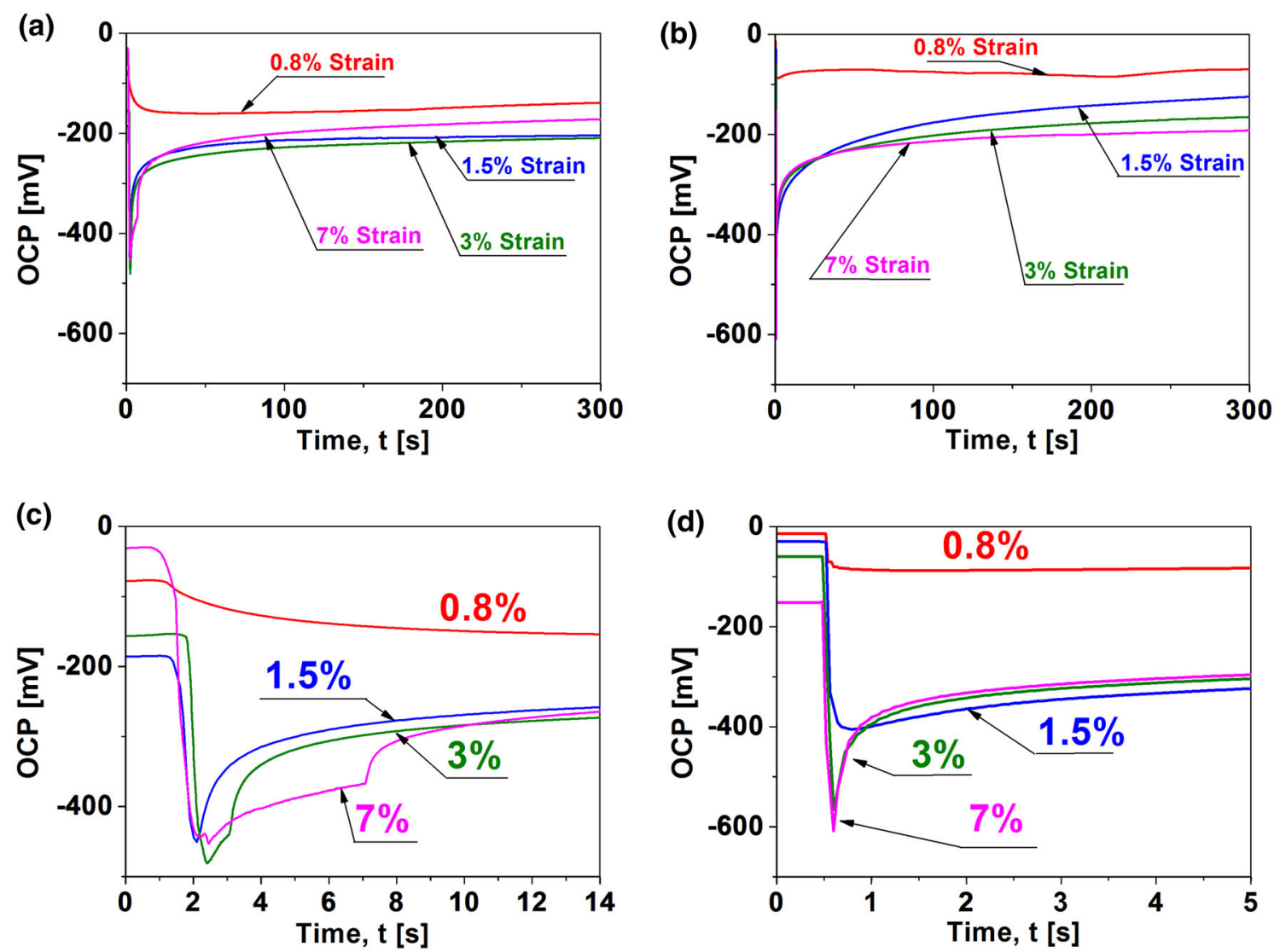

Fig. 5 Summary of OCP kinetics tests. Evolution of the OCP on NiTi wires deformed to various tensile strains $0.8,1.5,3$, and $7 \%$ using strain rate $=1 \% \mathrm{~s}^{-1}(\mathbf{a}, \mathbf{c})$, and $180 \% \mathrm{~s}^{-1}(\mathbf{b}, \mathbf{d})\left(\right.$ simulated biofluid, $\left.T=37{ }^{\circ} \mathrm{C}\right)$

mechanically induced damage to the surface layer as well as the progress of the subsequent chemical reactions. The chemical reactions proceed extremely fast once the surface oxide damage occurs and continue to proceed with gradually slowing down rate. Studying their kinetics in detail is essential for cyclic superelastic fatigue of NiTi wires, since the progress of the periodical surface oxide damage and passivation during tensile cycling cannot be understood without understanding the kinetics of the mechanically triggered chemical reactions.

\section{In Situ OCP Experiments During Cyclic Tensile Loading}

With this knowledge from kinetics tests, let us proceed to the in situ OCP studies of NiTi wire subjected to tensile cycling, where the damage and passivation processes occur periodically. The key point is that superelastic strains as large as $8 \%$ are reversible in cyclic tensile tests and the same of course is the case for surface strains. This means that reversible surface strains as large as $8 \%$ occur repeatedly during the cyclic tensile test. Not only the oxide layer fractures but also the subsurface layer of the NiTi matrix is periodically stressed, strained, and martensitically transformed from the austenite to martensite. All this potentially affects the mechanochemical processes at the wire surface.

Due to the nonlinear hysteretic character of the superelastic stress-strain response of $\mathrm{NiTi}$, we make a distinction between the elastic deformation in austenite state at small strains $(\Delta \varepsilon<1 \%$, "Cyclic Loading in Austenite Elastic Range" section), and superelastic deformation at larger strains $(\Delta \varepsilon=1-7 \%$, "Superelastic Cycling Involving Stress Induced Martensitic Transformation" section). Further on, we investigate the effect of strain rate, mean strain, strain amplitude, and temperature on fatigue performance. Let us, finally, note that the in situ OCP method is noninvasive in electrochemical sense.

\section{Cyclic Loading in Austenite Elastic Range}

As observed already in the kinetics experiments, OCP slightly decreases upon loading in austenite elastic range (Figs. 6a, 7a, b), where no shear band and hence no surface oxide cracking takes place. Recall that almost no OCP increase (passivation) was observed in tests with lowest 
(a)

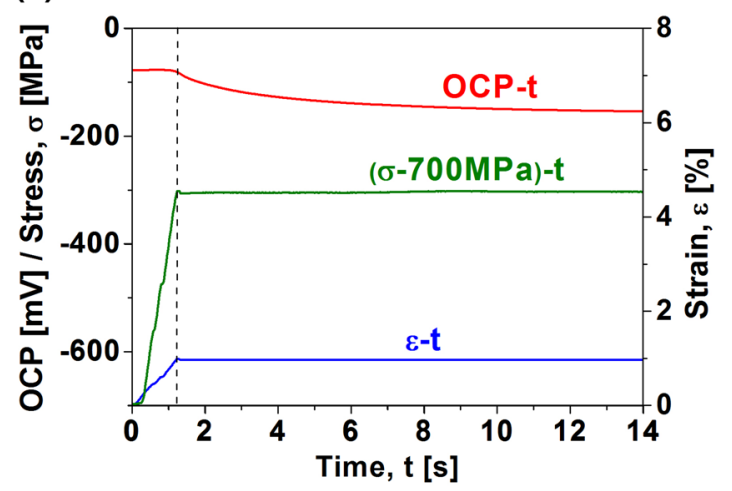

(c)

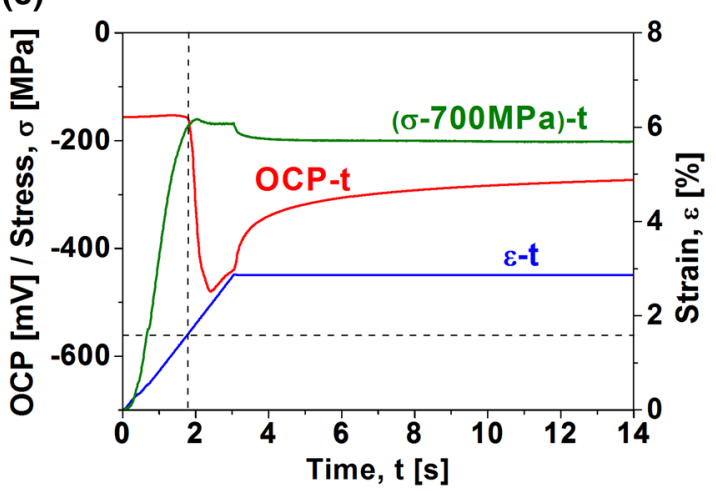

(b)

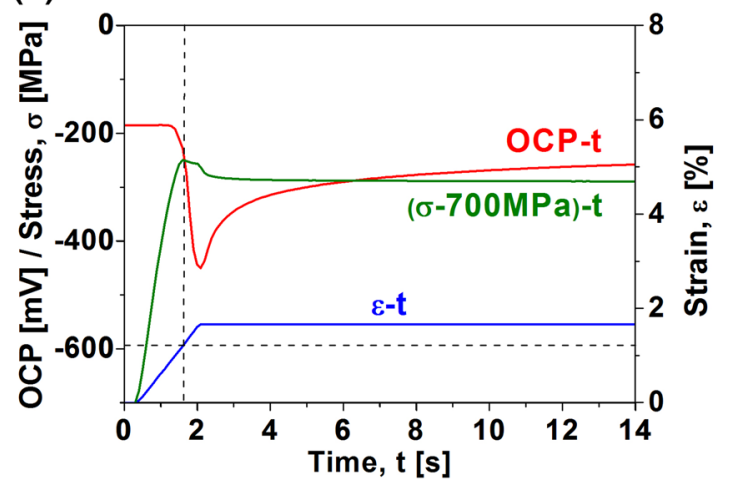

(d)

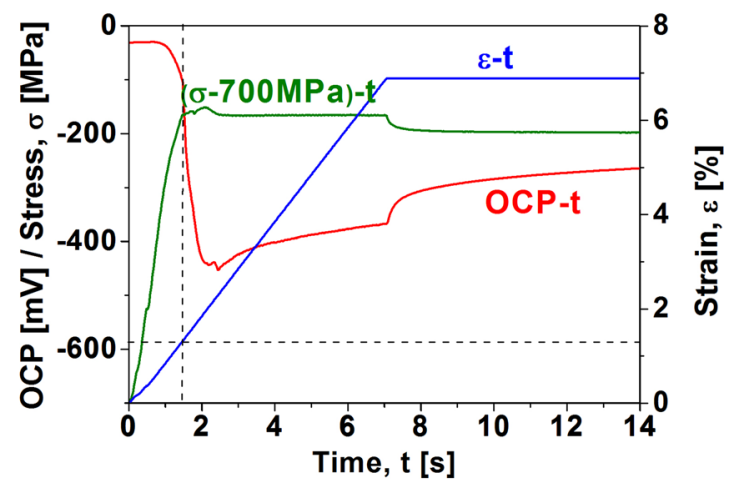

Fig. 6 OCP kinetics test, slow straining. Evolution of the strain, stress, and OCP at the onset of the kinetics tests (strain rate $=1 \% \mathrm{~s}^{-1}$, simulated biofluid, $T=37{ }^{\circ} \mathrm{C}$ ) for applied strains $1,1.7,2.9$, and $6.9 \%$. Dashed lines show the austenite elastic strain limit

applied strains. Figure 9 shows results of in situ OCP measurement during the cyclic loading of the NiTi wire in austenite state (Fig. 9a, b) (no oxide cracking in the first cycle assumed) and tungsten wire (Fig. 9c, d) for comparison. It can be seen that the OCP at first decreases in the first 30 cycles (NiTi) or 70 cycles (tungsten) and slightly increases (NiTi) or remains nearly constant (tungsten) upon cycling in later stages.

There are significant differences between the OCP responses of NiTi and tungsten. Neglecting the very different OCP values for both wires, which is most likely due to different surface oxides and different electrochemical reactions, main difference is that the NiTi shows much larger OCP variation during single cycle. Since the damage prevails over passivation in the first 30 cycles, the OCP gradually decreases upon cycling. Later on, however, the situation reverses and OCP gradually increases.

\section{Superelastic Cycling Involving Stress-Induced Martensitic Transformation}

Figure 10 shows in situ OCP response during the first cycle of the complete cyclic superelastic test. As the wire deforms in the first cycle, the OCP at first drops at around $1 \%$ strain to the value as low as $-450 \mathrm{mV}$ due to the oxide cracking but increases back up to $-300 \mathrm{mV}$ (Fig. 10c) in the rest of the first tensile cycle due to the massive passivation occurring simultaneously with the propagation of the shear band. Upon further cycling, the OCP varies periodically with passivation prevailing over the damage so that the OCP gradually increases with the increasing number of cycles.

It is essential to realize that the amplitude of the cyclic variation of the OCP is much larger during the superelastic cycling $(\sim 30 \mathrm{mV})$ compared to the elastic cycling $(\sim 2 \mathrm{mV})$. This reflects the fact that both the damage and subsequent passivation are much stronger in the complete superelastic cycling. Looking at the stabilized OCP response within single tensile cycle more closely (Fig. 11a), we find out that it is very well defined, reproducible, and changed very little from cycle to cycle. Only in the very last cycles prior the fracture, the OCP starts to gradually decrease as Fig. $11 \mathrm{~b}$ shows.

It shall be pointed out that, although the OCP value gradually increases upon cycling, the average value of the cycled wire remains low $(<-200 \mathrm{mV})$ compared to that of the static NiTi wire $(\sim-50 \mathrm{mV})$. The superelastically cycled NiTi wire in simulated biofluid is thus exposed for most of the lifetime to much more negative electrochemical potential than the static or elastically cycled NiTi wire. 
(a)

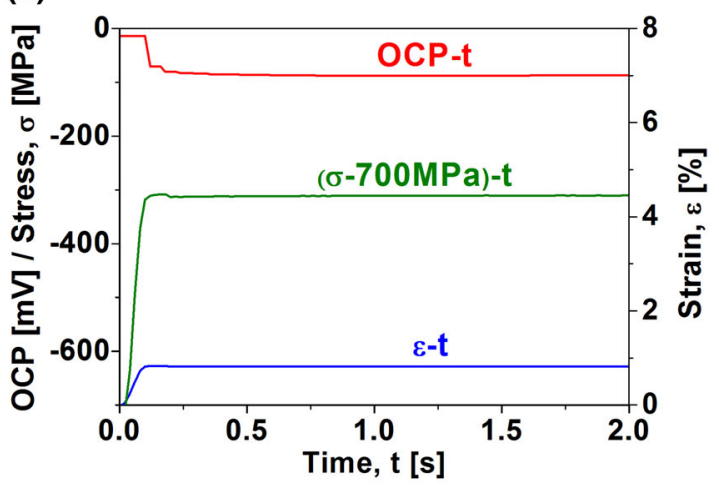

(c)

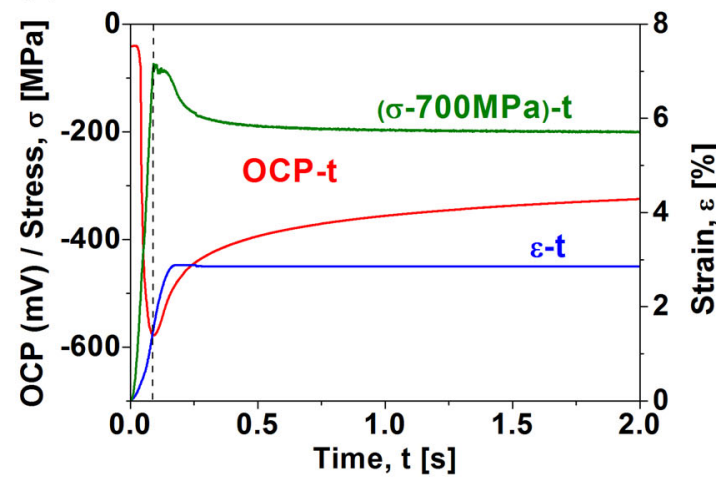

(b)

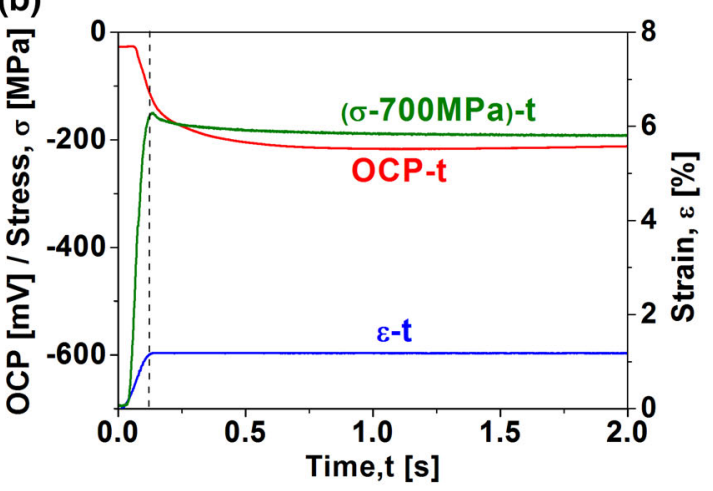

(d)

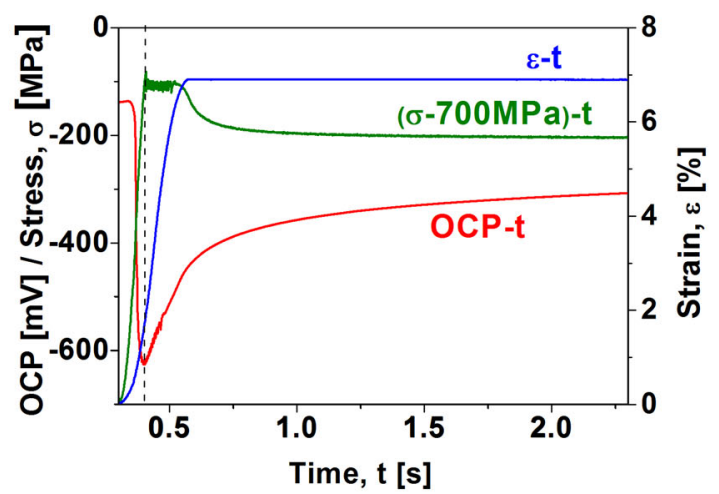

Fig. 7 OCP kinetics test, fast straining. Evolution of the strain, stress, and OCP at the onset of the kinetics tests $\left(\right.$ strain rate $=180 \% \mathrm{~s}^{-1}$, simulated biofluid, $T=37^{\circ} \mathrm{C}$ ) for applied strains $0.8,1.2,2.9$, and $6.9 \%$. Dashed lines show the austenite elastic strain limit

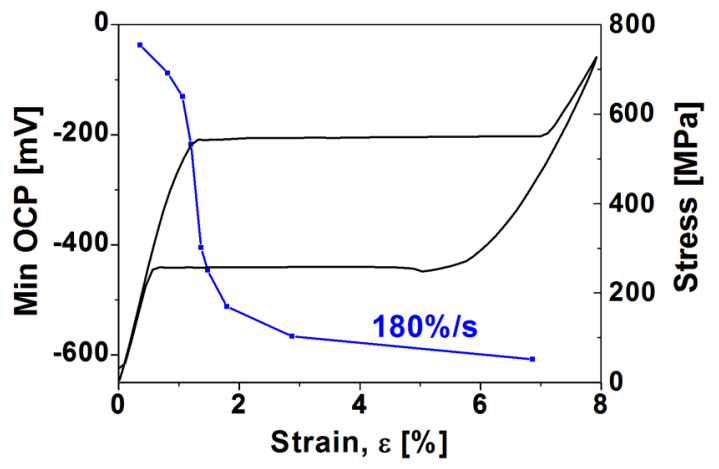

Fig. 8 Dependence of the minimum of the OCP on the applied strain observed in kinetics tests using strain rate $180 \% \mathrm{~s}^{-1}$ (Figs. 5, 7). Superimposed is superelastic stress-strain curve (strain rate $=3 \% \mathrm{~s}^{-1}$, simulated biofluid, $T=37^{\circ} \mathrm{C}$ )

Effect of Strain Rate Since the passivation of the damaged surface is a time-dependent process, we performed dedicated tests to find out how the OCP evolves with number of cycles at different strain rates (Fig. 12) and how the stabilized OCP response (Fig. 13) looks like in these tests. In order to assess the evolution of the OCP during the fatigue lifetime, we plot the "mean OCP" value recorded at the strain $\varepsilon=0$ in dependence on the number of cycles till fracture in Fig. 12. To show the OCP evolution at the beginning as well as at the end of tests, the overall mean OCP evolution during the fatigue lifetime is plotted in linear scale (Fig. 12a) as well as in logarithmic scale (Fig. 12b). Three tests were performed with each strain rate to achieve minimum statistics necessary to assess the effect of strain rate on the number of cycles till failure. Clearly, the maximum OCP level $\mathrm{OCP}^{\mathrm{MAX}}$ as well as the number of cycles till failure $\mathrm{N}^{\mathrm{F}}$ decreases with the increasing strain rate (Fig. 12c). On the other hand, transformation stress (Fig. 12d) increases with the increasing strain rate too. Figure 13 shows that the OCP varying during single superelastic cycle in the stabilized regime (100th cycle) decreases from $-150 \mathrm{mV}$ at the rate $0.5 \% \mathrm{~s}^{-1}$ down to $-275 \mathrm{mV}$ at the rate $10 \% \mathrm{~s}^{-1}$. The character of the OCP variation during the single superelastic cycle seems to be rather flat and rate independent.

These results suggest that the passivation of the surface damage is less effective when the cyclic test is carried out with higher strain rate. It might have an impact on the number of cycles till failure, on the other hand, the higher transformation stress at higher strain rates might affect the fatigue performance of the wire as well. 

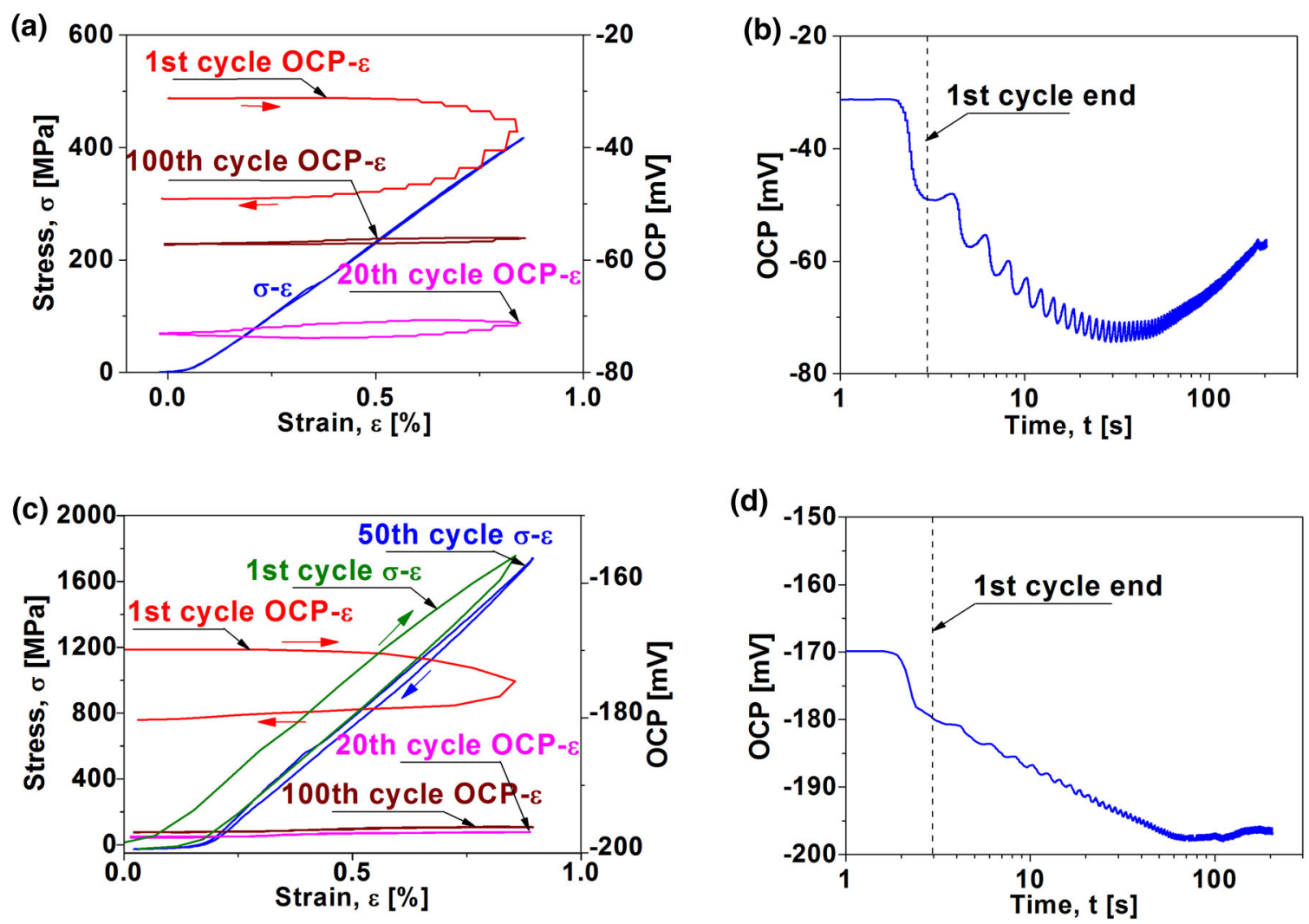

Fig. 9 Cyclic tensile test on NiTi $(\mathbf{a}, \mathbf{b})$ and Tungsten wire $(\mathbf{c}, \mathbf{d})$ in elastic range (mean strain $=0.4 \%$, strain amplitude $=0.4 \%$, strain rate $=1 \% \mathrm{~s}^{-1}$, simulated biofluid, $T=37^{\circ} \mathrm{C}$ ). Stress and OCP are

Effects of Mean Strain and Strain Amplitude Strain localization in shear bands represents a major problem for fatigue studies on NiTi wire in tension. Once the stress-induced martensitic transformation starts and shear band front(s) move during the cyclic tensile test, the strain on the wire surface approaches $7 \%$ even if the imposed strain amplitude is as small as $1 \%$. Figure 14 compares the results of three tests performed with small strain amplitude $0.5 \%$ at three different mean strains-in austenite state $(0.5 \%)$, in the mid of the transformation range $(4 \%)$, and in the martensite state $(7.5 \%)$. The shear band front does not exist in 0.5 and $7.5 \%$ cases. It exists but almost does not move during the cycle in case of the $4 \%$ mean strain test. It is found that the OCP recorded in 100th cycle increases with the increasing mean strain (Fig. 14b). Note, however, the OCP evolution upon cycling for different mean strains (Fig. 14c). It is qualitatively different for the three individual cases. The passivation of the surface damage proceeds very differently during the elastic loading in the austenite state and stressinduced martensite state. We did not wait for the OCP to increase after stretching the wire to $7.5 \%$ strain for the first time. If we would do that, the sharp OCP minimum on the corresponding curve in Fig. 14c would not most likely exist. plot in dependence on the strain $(\mathbf{a}, \mathbf{c})$ and time (b, d). Dashed lines in $\mathbf{b}$ and $\mathbf{d}$ denote the end of the first loading-unloading cycle

The number of cycles till failure is largest $\left(N^{\mathrm{F}}=16,000\right)$ when the test is performed at largest mean strain $7.5 \%$ in the martensite state and smallest $\left(N^{\mathrm{F}}=1050\right)$ for the mean strain in the transformation range at $4 \%$. This is very surprising taking into account that the stress applied during the tensile cycling in the martensitic state is by far highest (Fig. 14b). These tests were performed 3 times and the results are very well reproducible. The fatigue performance of the wire fatigued at the mean strain $4 \%$, where the shear band front moves, is worst.

Figure 15 compares the results of the three tests performed using the same mean strain $4 \%$ and three different strain amplitudes 1,2 , and $4 \%$. In contrast to the tests in Fig. 14, the shear band fronts move during the tensile cycling in all these tests. Note that due to the strain localization the surface strain varies from 1 to $7 \%$ as the shear band front moves upon cycling. It is found that the OCP at 100th cycle decreases with the increasing strain amplitude (Fig. 15b). The tests were again performed 3 times and the results are very well reproducible. The fatigue performance surprisingly improves with the increasing strain amplitude (Fig. 15c). 

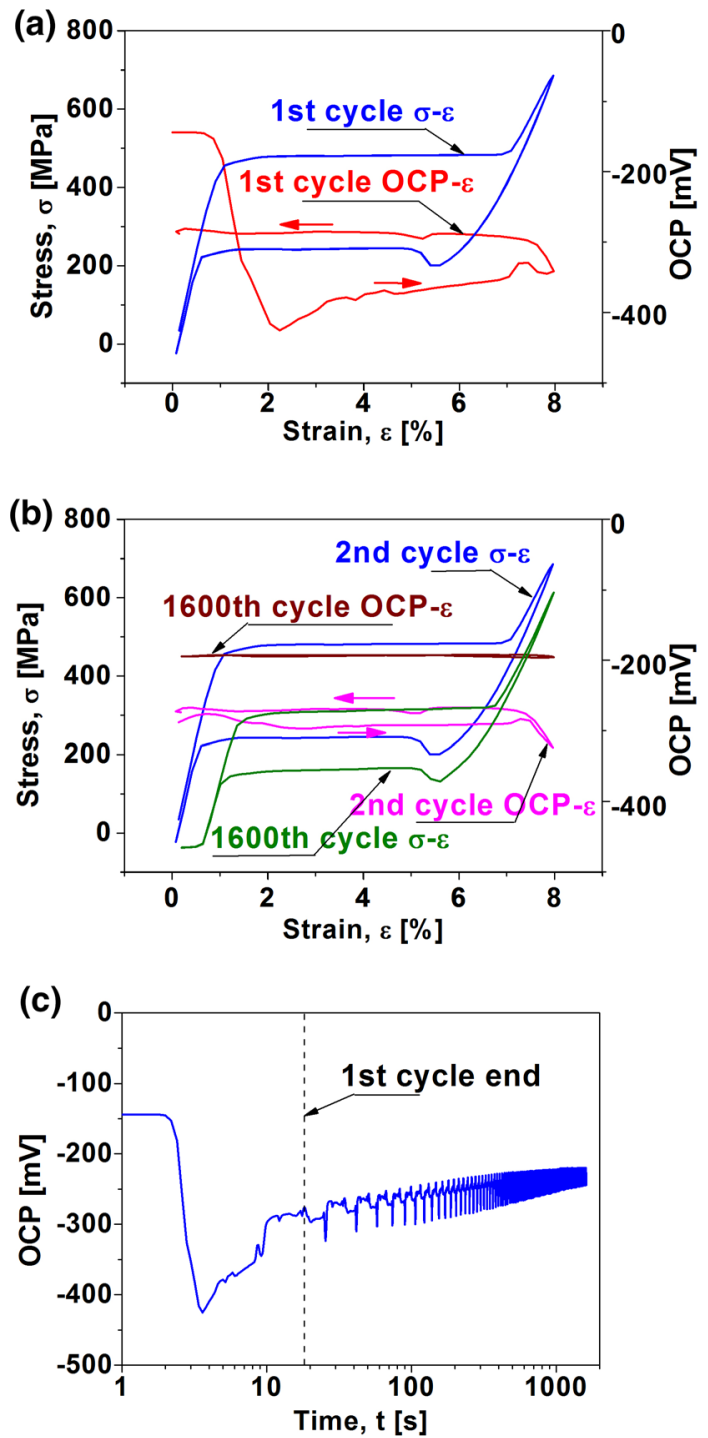

Fig. 10 Cyclic superelastic tensile test on $\mathrm{NiTi}$ wire (mean strain $=4 \%$, strain amplitude $=4 \%$, strain rate $=1 \% \mathrm{~s}^{-1}$, simulated biofluid, $T=37{ }^{\circ} \mathrm{C}$ ). Stress and $\mathrm{OCP}$ are plot in dependence on the strain (a, b) and time (c). The 1600th cycle corresponds to the maximum of the OCP $(\mathbf{c})$. The dashed line in $\mathbf{c}$ denotes the end of the first loading-unloading cycle

Effect of Temperature Since the temperature of the liquid may affect the passivation of the damaged surface as well as the fatigue lifetime, we performed experiments at different temperatures $27,37,40$, and $50^{\circ} \mathrm{C}$ (Fig. 16). Three tests were performed to achieve minimum statistics necessary to assess the effect of temperature on the number of cycles till failure. The mean $\mathrm{OCP}$ evolution during the fatigue lifetime is shown in linear scale (Fig. 16a) and logarithmic scale (Fig. 16b). The OCP seems to be independent on the temperature (Fig. 16c). What changes dramatically with the temperature is the forward transformation stress (Fig. 16d) and hysteresis width (Fig. 17). The pronounced effect of
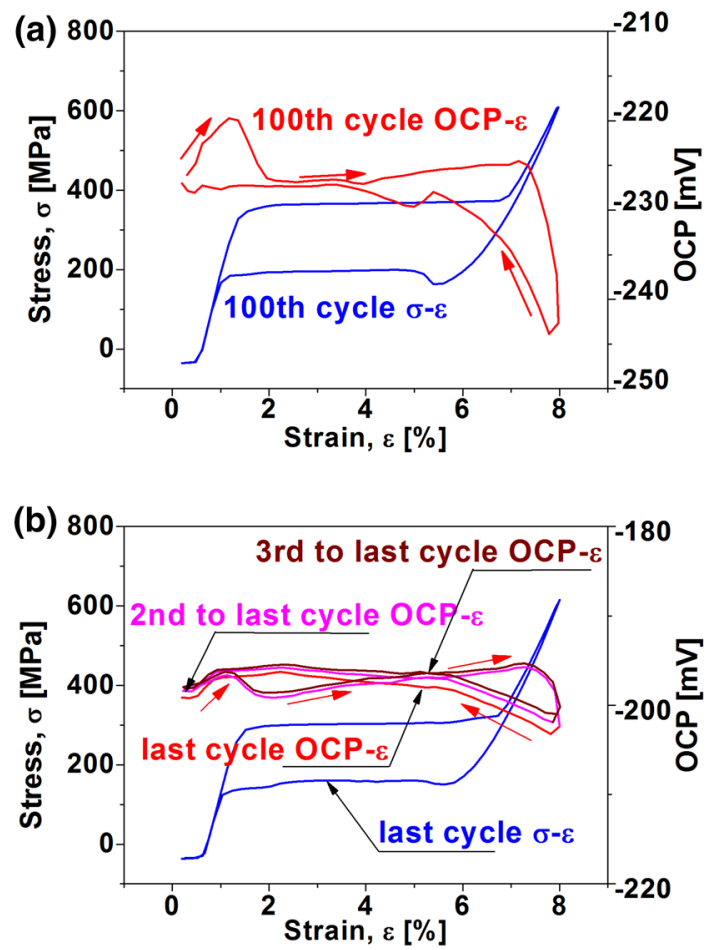

Fig. 11 Evolution of the stress and OCP response in the 100th superelastic cycle (a) and in the last cycles before fracture (b) in the superelastic test in Fig. 10 (mean strain $=4 \%$, strain amplitude $=4 \%$, strain rate $=1 \% \mathrm{~s}^{-1}$, simulated biofluid, $T=37^{\circ} \mathrm{C}$ )

increasing temperature on decreasing fatigue life (Fig. 16c) might be due to the increase of transformation stress with the increasing temperature.

\section{Damage Accumulation, Strength, and Failure}

All the results discussed so far seem to show that the OCP evaluated upon tensile cycling gradually increases till the fracture. However, looking closely at the evolution of the OCP in the latest stages of the tests, it can be seen that the OCP always reaches a maximum few tens (hundreds) of cycles before the final fracture (Fig. 18b). The occurrence of the OCP maximum on the OCP-N curve thus heralds the upcoming fracture. It is assumed to be connected to the accelerated growth of fatigue cracks into the bulk. The phenomenon has been already exploited in the field to asses fatigue lifetime of conventional metals [32]. Let us point out that the continuously recorded stress-strain-electric resistivity during the fatigue tests, reflecting the microstructure evolution in the bulk, provides no warning that the wire will break soon.

In view of this, the correlation of the OCP evolution with the degradation of the strength of the cycled NiTi wire was investigated (Fig. 18). When the OCP reached maximum (S1) or entered the decreasing stage beyond the 

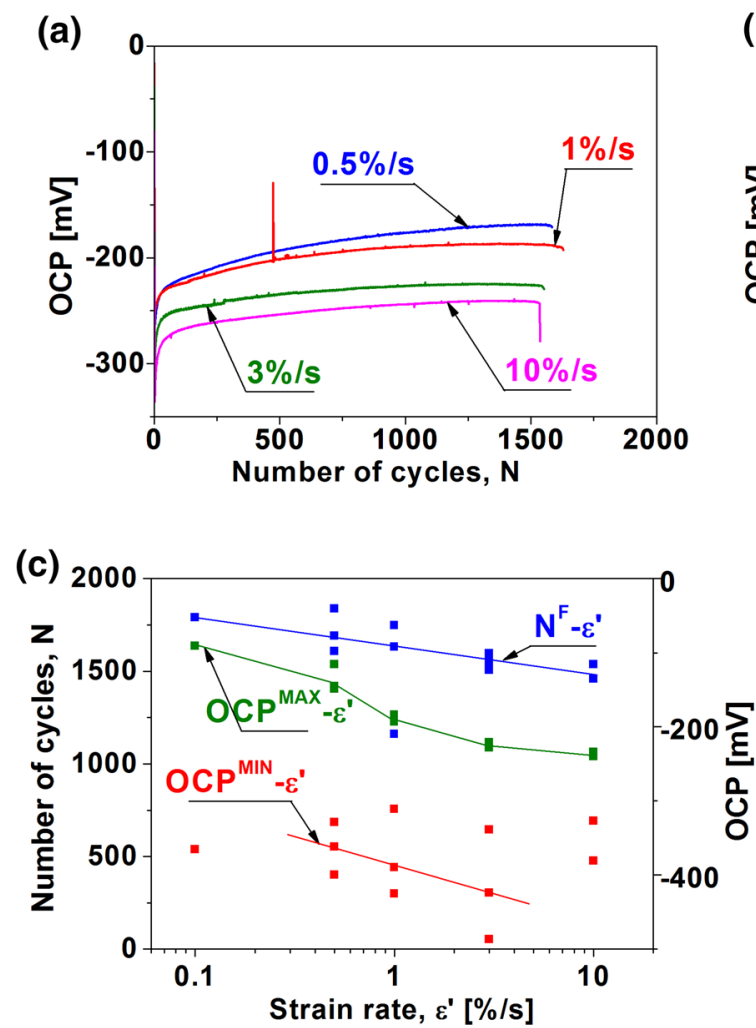

Fig. 12 Strain rate effect on fatigue. Evolution of OCP recorded at strain $\varepsilon=0$ during the tensile cycling using various strain rates $0.5,1$, 3 , and $10 \% \mathrm{~s}^{-1}$ (simulated biofluid, $T=37{ }^{\circ} \mathrm{C}$ ). Evolution of the OCP with number of cycles is shown in linear (a) and logarithmic

maximum (S2, S3), the cyclic tests were stopped and the wires were loaded in tension up to the fracture. It was found that the strength did not change with the increasing number of cycles for most of the fatigue lifetime. After the OCP approached the maximum, the strength decreased rapidly (Fig. 18a, c). This shows that the OCP decreasing after reaching the maximum correlates with the rapid deterioration of the strength of the wire.

In order to confirm that the OCP maximum indeed corresponds to the accumulating surface damage, we have investigated the surface of the wire cycled till the saturation by SEM (Figs. 19, 20). As mentioned earlier, the oxide cracks had to be observed on a wire stretched in tension since they close and effectively disappear upon unloading. See the same place on the wire surface observed under stress and stress free in Fig. 20. The minor cracks are homogeneously distributed over the wire surface, oriented perpendicularly to the tensile load axis and mutually separated by $\sim 500 \mathrm{~nm}$ (Figs. 19, 20). Compared to the first cycle (Fig. 1), in addition to the small cracks which remain similar, there are also few longer and wider cracks that newly appeared during the cyclic loading. These longer cracks are about $10 \mu \mathrm{m}$ long and $1 \mu \mathrm{m}$ wide and always nucleate at the inclusions and notches (Fig. 19).
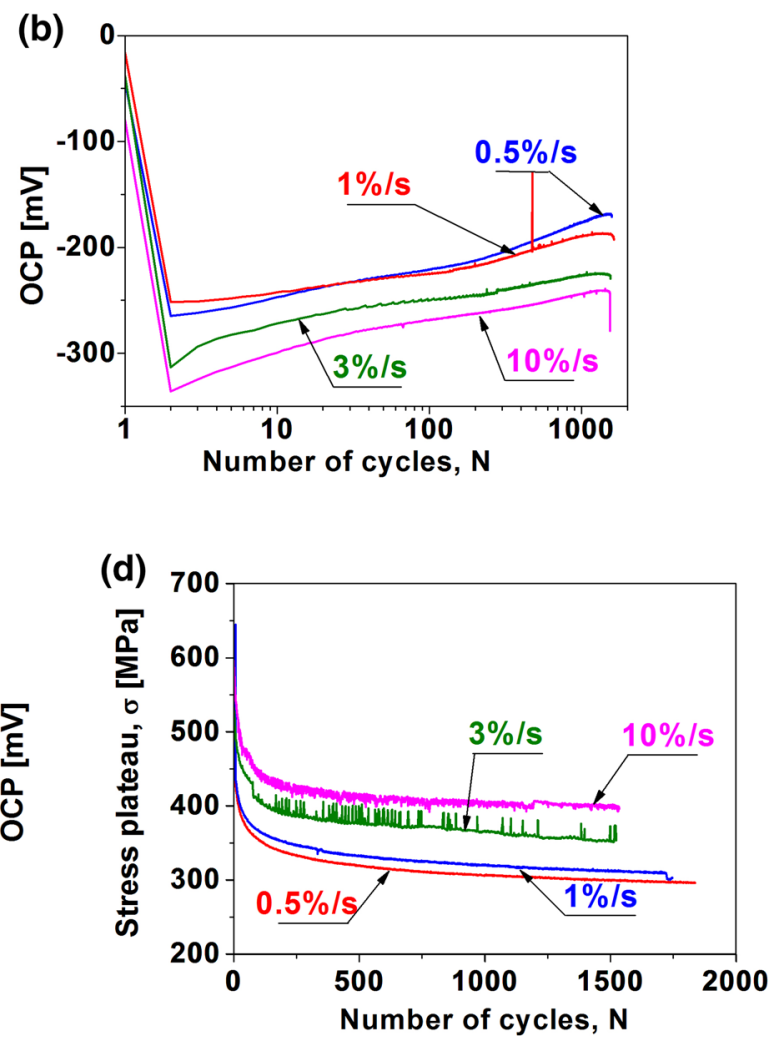

(b) scales. Strain rate dependences of the minimum $\mathrm{OCP}^{\mathrm{MIN}}$ and maximum $\mathrm{OCP}^{\mathrm{MAX}}$ values and number of cycles till failure $N^{\mathrm{F}}$ are presented in c. Evolution of the forward transformation stress with number of cycles is shown in $\mathbf{d}$

This confirms that the accelerated growth of fatigue cracks into the NiTi matrix is indeed related to the OCP decrease. The OCP is very sensitive to the surface damage. Because of that, it heralds the upcoming wire fracture even if the stress-strain-electrical resistance response fails in that respect.

No detachment of the oxide layer, oxide flaking, or any other oxide damage induced by tensile cycling was ever observed.

An appealing question was whether the tiny oxide cracks serve as nucleation sites for fatigue cracks growing later upon cycling into the NiTi matrix. To find an answer, FIB cross sections perpendicularly to the larger cracks were observed by SEM (Fig. 20c-e). A dense array of short matrix cracks (typically 100-300 nm long and $\sim 500 \mathrm{~nm}$ separated) was observed on the cross sections right under the surface oxide layer. Major cracks growing several microns deep into the NiTi bulk were observed underneath the major surface cracks. A one-to-one correlation between the tiny oxide cracks and short matrix cracks was clearly confirmed (Fig. 22). The cracks were also studied in detail by $3 \mathrm{D}$ reconstruction technique by automated FIB milling combined with SE scanning. The results will be reported separately. 

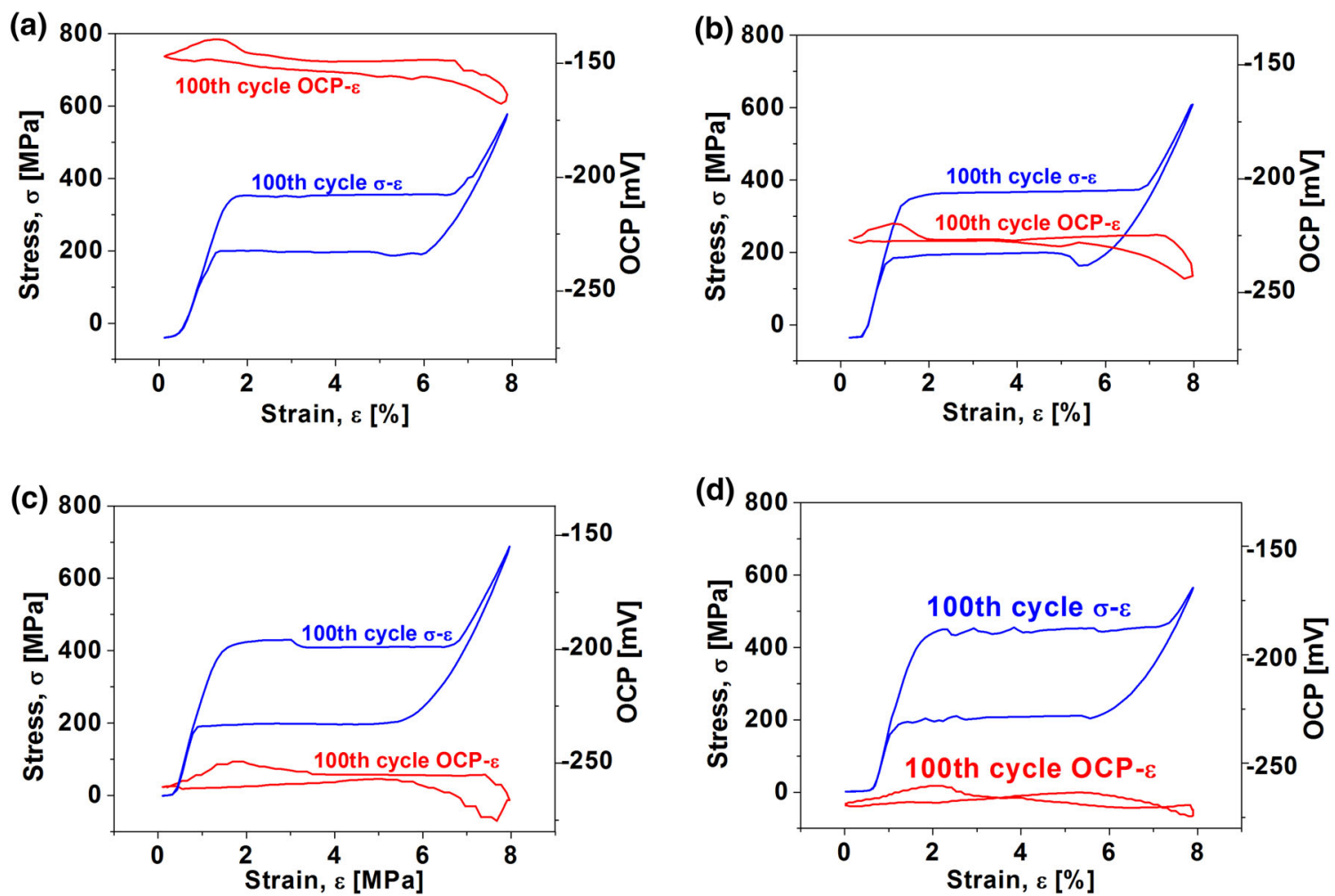

Fig. 13 Evolution of the stress and OCP response in the 100th superelastic cycle from cyclic tensile tests (Fig. 12) performed using strain rates a $0.5 \% \mathrm{~s}^{-1}, \mathbf{b ~} 1 \% \mathrm{~s}^{-1}$, c $3 \% \mathrm{~s}^{-1}$, and $\mathbf{d} 10 \% \mathrm{~s}^{-1}$ (mean strain $=4 \%$, strain amplitude $=4 \%$, simulated biofluid, $T=37{ }^{\circ} \mathrm{C}$ )

\section{Discussion}

Corrosion properties of NiTi immersed in biofluids have been studied since the very beginning of the development of superelastic technologies. Overwhelming literature evidence supports the view that carefully engineered $\mathrm{TiO}_{2}$ surface oxide layer provides excellent protection to static NiTi implants immersed in biofluids [18-20] and reasonable protection to $\mathrm{NiTi}$ implants deforming elastically in biofluids $[12,19]$. The results presented above suggest that the situation might be very different in case of NiTi implants that deform superelastically in body fluids.

\section{Cracked $\mathrm{TiO}_{2}$ Oxide}

When NiTi deforms superelastically in liquids, the ceramic oxide layer on its surface cannot withstand the large superelastic strain accompanying propagation of the shear band and fractures in the very first tensile cycle (Fig. 1). Following that, the surface oxide contains array of regularly spaced nanocracks arranged perpendicularly to the wire axis. The surface of superelastically cycled NiTi wire is thus covered not by the surface oxide but by the "cracked surface oxide." The density (separation) of the oxide cracks is assumed to correlate with the plateau strain, though no specific investigations were carried out.
When shear band transformation proceeds in the cyclic superelastic tensile test, the surface deformation changes periodically from $\sim 1$ to $\sim 7 \%$ at the moving shear band front. The damage of the already cracked surface oxide layer upon cycling is assumed to be limited to the existing oxide cracks. The stress in the oxide areas among the existing cracks is expected to be lower than it was at the peak elastic stress in the first cycle, which is why it does not fracture anymore upon tensile cycling and why the small cracks (length, density) look similar after the 1st cycle and 1500th cycle. The damage introduced into the surface in each superelastic cycle is thus assumed to be localized into the tips of the existing oxide cracks.

Film rupture problems related with corrosion of strained metals have been thoroughly studied in the past, particularly to the aqueous stress corrosion cracking of reactor steels. The models developed in the literature deal with film rupture accelerated corrosion [30] of deforming metals. They assume that straining creates electrochemically active surface simply by exposing fresh metal surface to the corrosive liquid. The current density of a partially activated surface (scales with the corrosion rate) is expressed as a product of the current density of the active surface and the activated area fraction $A^{*}$. The rate of change of $\mathrm{A}^{*}$ has been introduced [30] in a differential form (Eq. 4) as being due to the surface passivation rate 

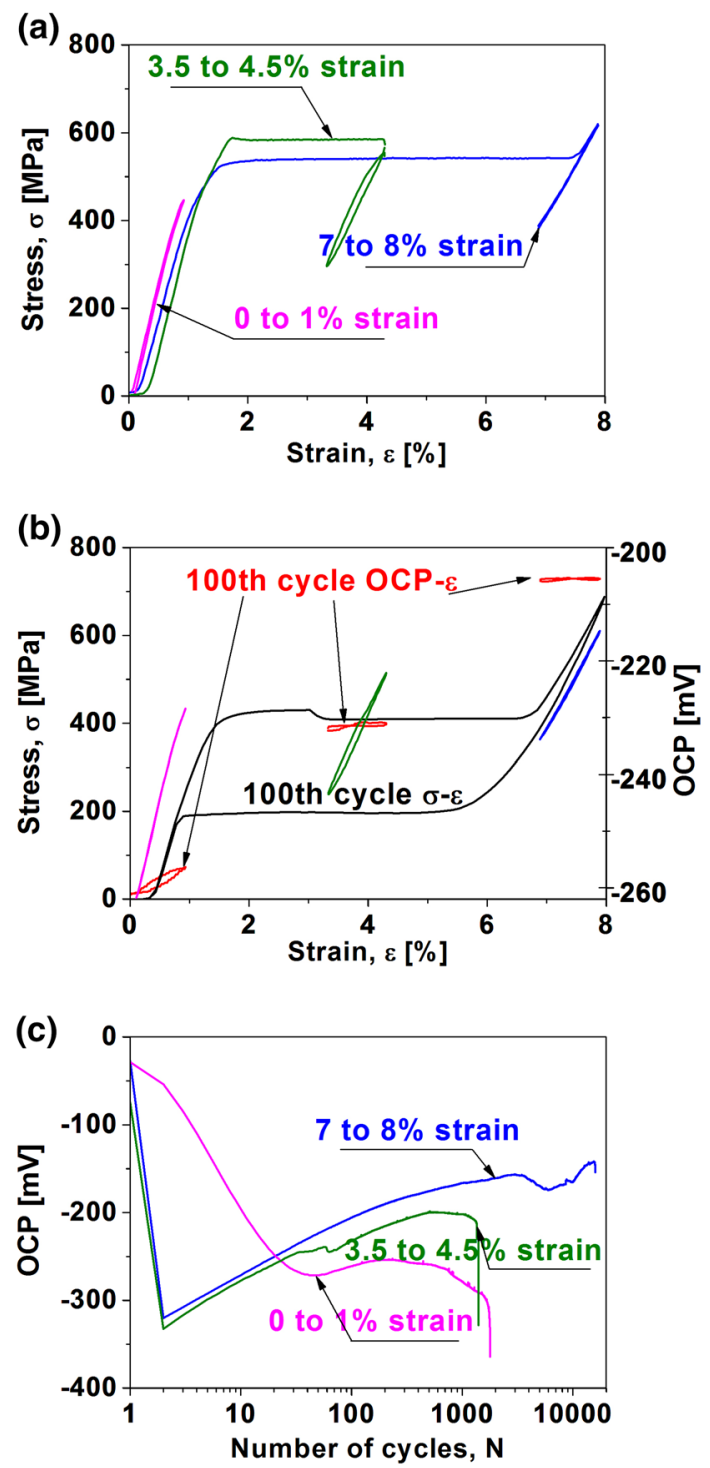

Fig. 14 Effect of the mean strain on fatigue. Evolution of stress and OCP during the tensile cycling with mean strains $0.5,3$, and $7.5 \%$. (strain amplitude $0.5 \%$, strain rate $=3 \% \mathrm{~s}^{-1}$, simulated biofluid, $T=37^{\circ} \mathrm{C}$ ). a Stress-strain curves in the 1 th cycle, $\mathbf{b}$ partial stressstrain and OCP-strain curves in the 100th cycle (the complete superelastic curve in 100th cycle is shown for comparison), c evolution of the OCP at strain $\varepsilon=0$ upon tensile cycling

due to either dissolution of active sites or growth of a protective film (left side term) acting against the straininduced $(\mathrm{d} \varepsilon / \mathrm{d} t)$ surface activation rate (right side term). It is assumed that rate with which the active surface fraction is passivated is proportional to the current area of active surface $\left(k^{*}\right.$ is the reference surface passivation rate). $0<\gamma<1$ is the active surface area fraction per unit strain - a constant that quantifies strain-based surface activation rate.
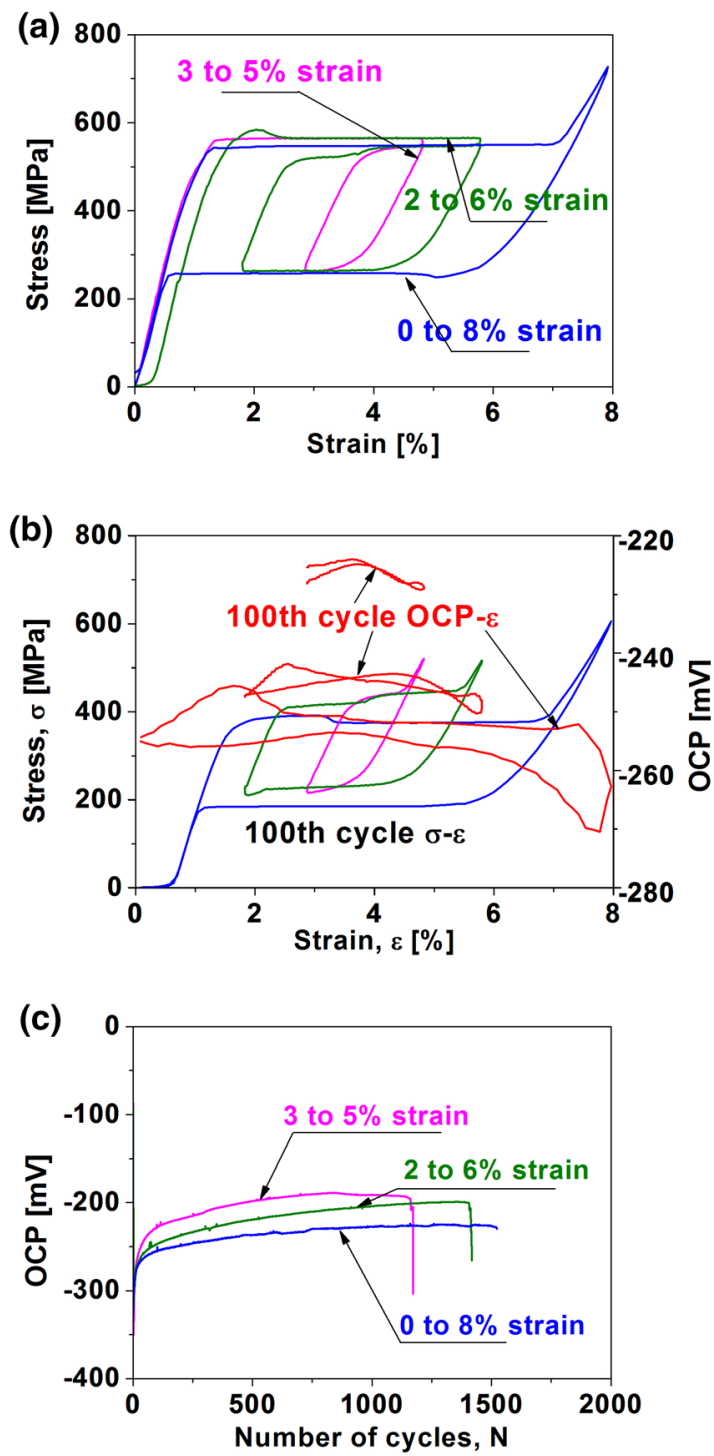

Fig. 15 Effect of the strain amplitude on fatigue. Evolution of the stress and OCP during the tensile cycling using strain amplitudes 1,2, and $4 \%$ (mean strain $=4 \%$, strain rate $=3 \% \mathrm{~s}^{-1}$, simulated biofluid, $T=37{ }^{\circ} \mathrm{C}$ ). a Stress-strain curves in the 1 th cycle, $\mathbf{b}$ partial stress-strain and OCP-strain curves in the 100th cycle, c evolution of the OCP at strain $\varepsilon=0$ upon tensile cycling

$\frac{\mathrm{d} A *}{\mathrm{~d} t}=\left(\frac{\delta A *}{\delta t}\right)_{\varepsilon}+\left(\frac{\delta A *}{\delta \varepsilon}\right)_{t} \frac{\mathrm{d} \varepsilon}{\mathrm{d} t}=-k * A *+(1-A *) \gamma \frac{\mathrm{d} \varepsilon}{\mathrm{d} t}$

Although, the actual chemical reactions occurring at the fresh surface of reactor steel and NiTi are different, similar approach is currently being developed for environmental fatigue of NiTi, taking into account the strain reversibility-i.e., that the activated area fraction $A^{*}$ is up to some extent reversible upon unloading. The results will be reported separately. 

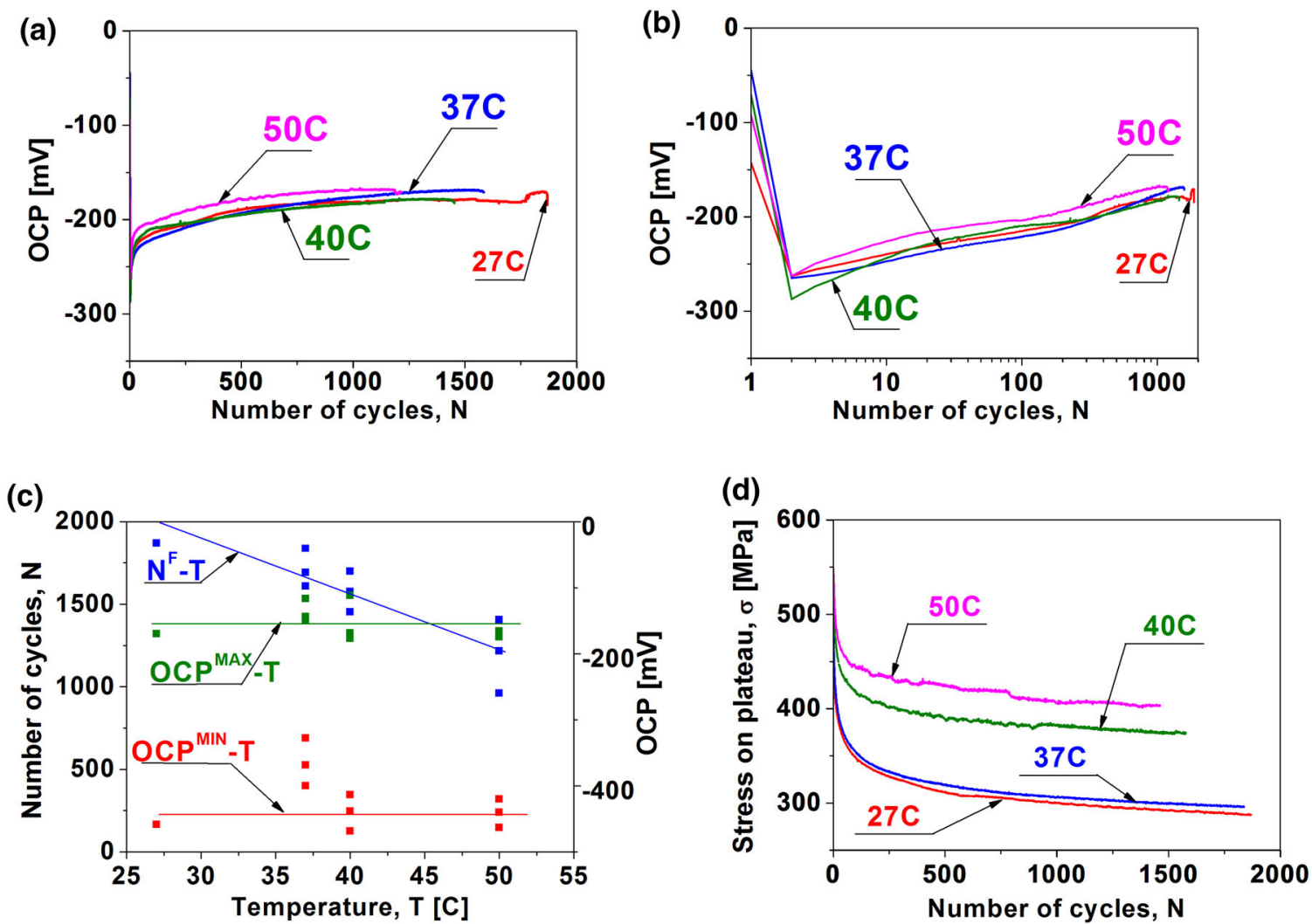

Fig. 16 Effect of temperature on fatigue. Evolution of the OCP recorded at strain $\varepsilon=0$ during the tensile cycling at temperatures $T=27,37,40,50{ }^{\circ} \mathrm{C}$ (mean strain $=4 \%$, strain amplitude $=4 \%$, strain rate $=0.5 \% \mathrm{~s}^{-1}$, simulated biofluid). Evolution of the OCP recorded at strain $\varepsilon=0$ during tensile cycling is shown in linear

\section{Mechanochemical Reactions at the NiTi Surface}

As introduced in "Experimental Methods" section, chemical reactions yielding electrons (Eq. 1) and atomic hydrogen (Eq. 2) proceed when NiTi wire is immersed into the simulated biofluid. Electrochemical quasiequilibrium characterized by the Open Circuit Potential is established after a period of time. The OCP experimentally measured in experiments reflects the kinetics of both half-cell reactions for given state of the surface-i.e., it corresponds to minimum current density in Potentiodynamic test shown in Fig. 21a. Since the NiTi is protected by $\mathrm{TiO}_{2}$ oxide layer (passivated), the activity of these reactions on static NiTi implant immersed in biofluids is very limited (Fig. 21b), which gives rise to excellent corrosion resistance of the static NiTi [18].

In reality, the $\mathrm{TiO}_{2}$ surface oxide is never ideal, there are inhomogeneities, notches, inclusions, etc., where the chemical reactions do proceed and hence the OCP is different from zero. Since the negative potentials prevail in case of the NiTi, cathodic current dominates the cell current and hence OCP levels typically approach slightly (a) and logarithmic (b) scale. Temperature dependences of the minimum $\mathrm{OCP}^{\mathrm{MIN}}$ and maximum $\mathrm{OCP}^{\mathrm{MAX}}$ values and number of cycles till failure $N^{\mathrm{F}}$ are shown in c. Evolution of the forward transformation stress at $4 \%$ strain with number of cycles is shown in $\mathbf{d}$

negative values $(\sim-50 \mathrm{mV}$ in case of heat-treated $\mathrm{NiTi}$ with thicker oxide (Fig. 21b) and $\sim-200 \mathrm{mV}$ in case of mechanically polished NiTi wire with thinner oxide). Note that there is an anomaly on the Potentiodynamic polarization curve of the oxide-free wire around $0 \mathrm{mV}$ (Fig. 21a). This is assumed to be due to the massive oxidation reaction taking place upon increasing the potential in the test on the mechanically polished wire in which the reactions are controlled by the externally applied potential.

The stable electrochemical situation at the passivated surface of the NiTi wire immersed for about an hour changes dramatically when the surface oxide is mechanically damaged by loading the wire and the electrolyte comes into contact with the bare NiTi surface. Even slightest deformation of the metal may introduce nanocracks into the brittle surface oxide which instantaneously affects the chemical reactions (Eqs. 1, 2), particularly, the anodic reaction (Eq. 1) massively releases free electrons into the NiTi metal and shifts the potential of the NiTi alloy to more negative values in response to the damage. The electrochemical cell reacts by instantaneous change of the OCP potential to more negative values. The negative 

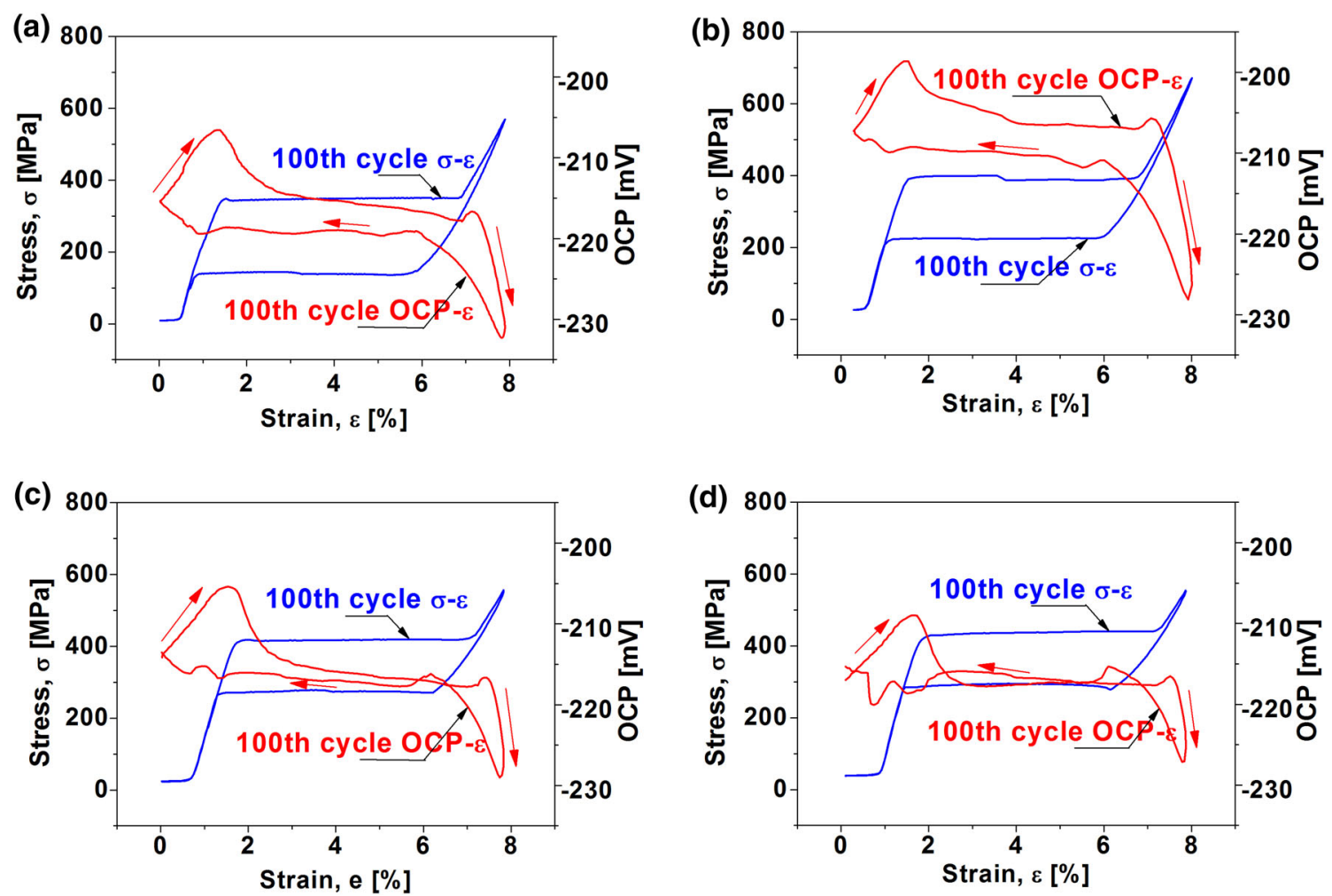

Fig. 17 Evolution of the stabilized stress and OCP response of the NiTi wire in the 100th superelastic cycle from cyclic tensile tests (Fig. 16) at temperatures: $\mathbf{a} T=27{ }^{\circ} \mathrm{C}, \mathbf{b} 37^{\circ} \mathrm{C}, \mathbf{c} 40{ }^{\circ} \mathrm{C}$, d $50{ }^{\circ} \mathrm{C}$ (mean strain $=4 \%$, strain amplitude $=4 \%$, strain rate $=0.5 \% \mathrm{~s}^{-1}$, simulated biofluid)

potential in turn slows down the anodic reaction and speeds up the cathodic reaction (passivation), which is the reduction of hydrogen ions $\mathrm{H}^{+}$giving rise to atomic hydrogen. As a result, the OCP increases back to the more positive (noble) values.

This would be the general scenario. NiTi, however, is somehow special. Separately, both $\mathrm{Ni}$ and Ti have different electrochemical behaviors. According to the nobility classification of metals [33], $\mathrm{Ni}$ is more noble than $\mathrm{Ti}$ (Fig. 21a). However, equiatomic NiTi alloy, similarly as $\mathrm{Ti}$, forms protective titanium oxides (Fig. 21b) which provide these metals with excellent protective passivation ability in contact with aggressive environments. In chemical terms, titanium is passivated by galvanic coupling with nickel. If cathode (Ti) and anode (Ni) area ratio is $1: 1$, exchange current dramatically decreases. The mixed potential of the Ni-Ti galvanic couple is thus even more noble than that of the Ti (Fig. 21a). Hence, NiTi alloys immersed in liquid environments with the concentration of $\mathrm{H}^{+}$are even more easily passivable than the unalloyed $\mathrm{Ti}$ and belong, because of this, among the noblest metals.

In acid solutions, the reaction governing the hydrogen evolution is given be Eq. 5. The initial step is the discharging process of the hydrated proton existing in the electrolyte. This reaction is more active at the excess electron region than at the deficient electron region on the electrode surface
(Fig. 22). In other words, electronegative Ni sites are more favorable than electropositive Ti sites for the cathodic discharging reaction and, consequently, nickel saturates titanium by cathodic current necessary for efficient oxidation. Recall that the oxidation affected Ni-rich subsurface lattice might be chemically inhomogeneous on the nanoscale giving rise to the $\mathrm{Ni}$ and $\mathrm{Ti}$ sites as suggested in Fig. 22. Nickel takes over the reducing function from the titanium. This " $\mathrm{Ni}+\mathrm{Ti}$ synergy" allows for extremely quick passivation (oxidation) of the newly opened bare NiTi surface (Eq. 6). At the same time, it leads to hydrogen overpotential and consequently to the high degree of the irreversibility of the hydrogen evolution via accelerated reaction (5).

$2 \mathrm{H}_{3} \mathrm{O}^{+}+2 \mathrm{e}^{-} \rightarrow \mathrm{H}_{2}+2 \mathrm{H}_{2} \mathrm{O}$

$\mathrm{Ti}^{2+}+2 \mathrm{H}_{2} \mathrm{O} \rightarrow \mathrm{TiO}_{2}+4 \mathrm{H}^{+}+2 \mathrm{e}^{-}$.

As explained above, the principle of galvanic passivation of NiTi is the cathodic reduction of hydrogen to a level stabilized by the newly formed passive oxide film that is essentially inert in the acid solutions. This newly formed surface oxide is again damaged by the excessive deformation in the next tensile cycle, the passivation proceeds via the oxidation reaction (Eq. 6) until the damage area is again passivated and this repeats cyclically. This newly formed passive film can be imagined as a gel consisting of hydrated dioxide $\mathrm{TiO}_{2} \cdot \mathrm{H}_{2} \mathrm{O}$ 

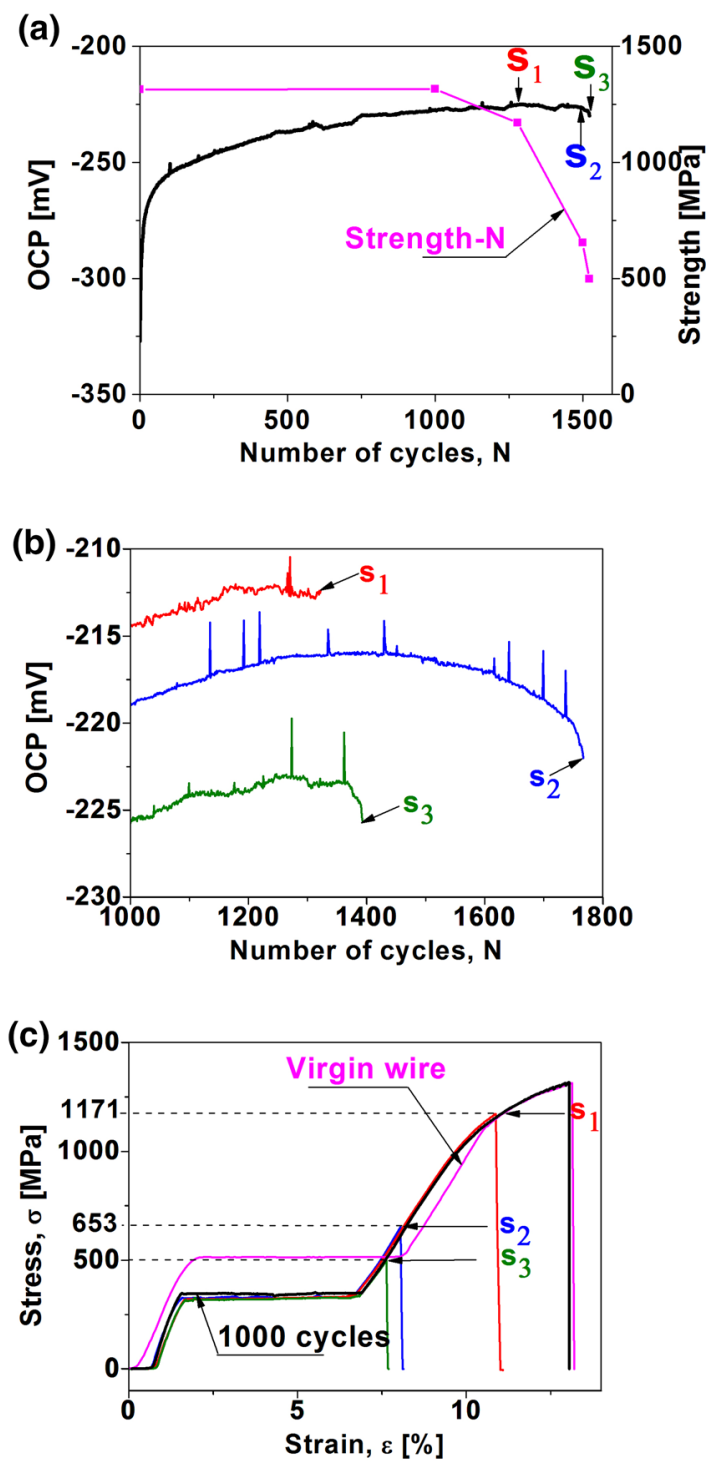

Fig. 18 Strength of the virgin and fatigued NiTi wires (mean strain $=4 \%$, strain amplitude $=4 \%$, strain rate $=3 \% \mathrm{~s}^{-1}$, simulated biofluid, $T=37^{\circ} \mathrm{C}$ ). The fatigue tests were stopped when the in situ evaluated OCP reached its maximum $\mathrm{OCP}^{\mathrm{MAX}}(\mathrm{S} 1)$ or even later when it was already decreasing (S2,S3), as suggested in a,b. The wires were subsequently deformed till fracture to evaluate their strength (c)

that fills the opening/closing oxide cracks tips (Figs. 22, 23) which can at least partially resist the repeated deformation. Since the OCP is increasing upon tensile cycling, we conclude that passivation ability of the hydrated dioxide gel gradually increases during the fatigue test.

\section{Hydrogen Uptake and Transport by the Cyclically Deformed NiTi}

Because of the high values of its diffusion coefficient, hydrogen can relatively easily move through the NiTi

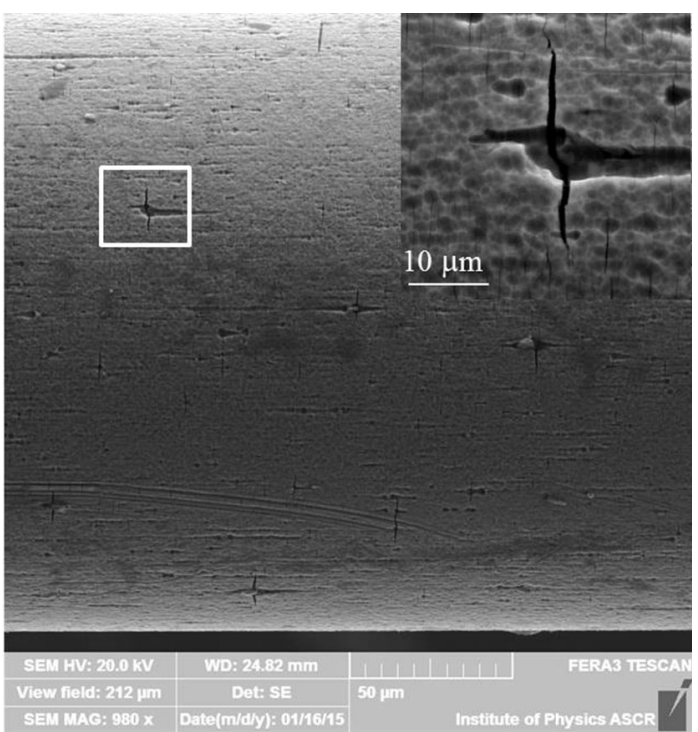

Fig. 19 SEM observation of the surface oxide cracks on the NiTi wire fatigued till $N=1500$ cycles $\left(\mathrm{OCP}^{\mathrm{MAX}}\right)$ in superelastic test (mean strain $=4 \%$, strain amplitude $=4 \%$, strain rate $=3 \% \mathrm{~s}^{-1}$, simulated biofluid, $T=37{ }^{\circ} \mathrm{C}$ ). The wire was kept in the elongated state during the SEM observation. While the small oxide cracks (detail in the inset) which are homogeneously dispersed over the wire surface cannot be seen in low magnification, the longer/wider oxide cracks appearing preferentially at inclusions and/or notches are clearly visible

lattice even at $37^{\circ} \mathrm{C}$ and just as easily be removed from it [34]. As the diameter of a hydrogen atom is very small, it occupies the interstitial sites at room temperature. As in other titanium alloys, hydrogen in NiTi also tends to form titanium hydrides [35]. Since the specific volume of titanium hydride is larger compared to that of the NiTi lattice, stress concentrations arise around it. NiTi can be hydrogenated either by exposing it to hydrogen atmosphere at high temperatures or by charging it electrolytically at room temperature. Virgin $\mathrm{TiO}_{2}$ layer prevents hydrogen from entering the NiTi from the electrolyte. When the surface oxide fractures, NiTi can be hydrogenated electrolytically even more easily. The hydrogen absorbed by the NiTi, however, suppresses the martensitic transformation [36]. In superelastically transforming $\mathrm{NiTi}$, suppression of the transformation inevitably leads to the increase of the local stress, plasticity or possibly even to cracking. According to the literature [37], the effect of absorbed interstitial hydrogen on the kinetics of dislocation and transformation processes is confined to a narrow temperature and strain rate windows, outside of which, solute hydrogen can have very different, even opposite effects.

Yokoyama and coworkers systematically investigated embrittlement of hydrogenated NiTi (see [38-40] and references there). They hydrogenated NiTi both by exposing it to gaseous hydrogen at high temperatures and by 
Fig. 20 SEM observation of the oxide cracks on the NiTi wire fatigued till $N=1500$ cycles in superelastic test (mean strain $=4 \%$, strain amplitude $=4 \%$, strain rate $=3 \% \mathrm{~s}^{-1}$, simulated biofluid, $T=37{ }^{\circ} \mathrm{C}$ ). Same place on the wire surface (f) is observed while keeping the wire in the shear band by the applied stress (a) and after unloading (b). Note that the closed surface oxide cracks in unloaded state (b) can be hardly resolved by SEM. The longer/wider cracks propagating deep into the $\mathrm{NiTi}$ matrix are clearly seen on FIBed wire cross sections (c) cut in places denoted on the surface perpendicularly to the cracks. Crack 1 (e) which nucleated at an inclusion extends deeper into the NiTi matrix than crack 2

(d) which nucleated at the notch
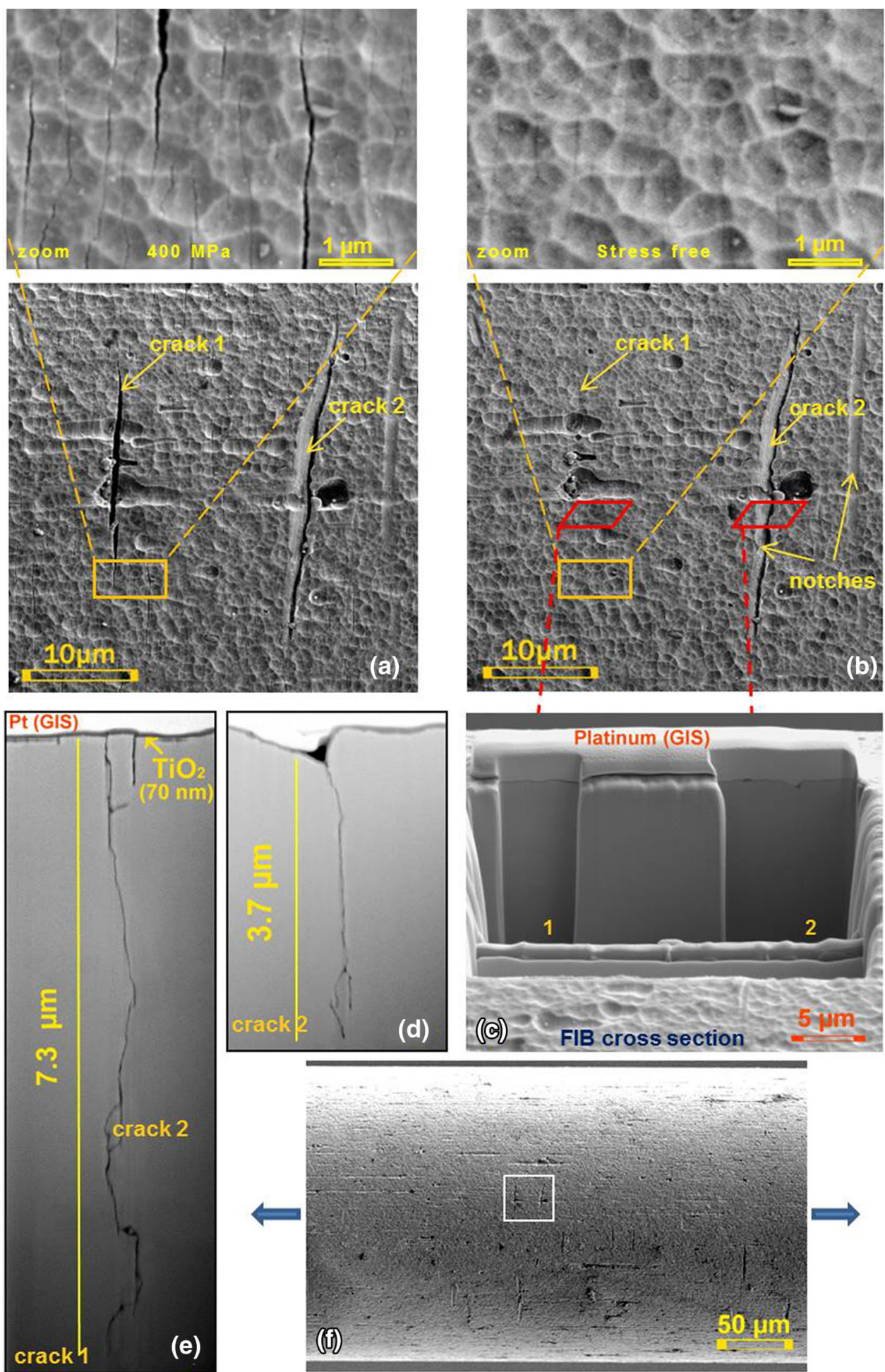

the electrolytic route to find out that the adsorbed hydrogen brings about severe decrease of the strength $[37,38]$ and leads to brittle fracture $[38,41]$. Particularly, they claimed that the hydrogen adsorption rate is much faster, when the NiTi is electrolytically hydrogenated while it transforms during cyclic mechanical loads [40], compared to the cases when it is stress free, under constant stress or cyclically loaded in elastic range.

Although the actual mechanism of the hydrogen embrittlement of NiTi needs to be investigated further, there should be no doubt that both the suppression of the martensitic transformation [36] and the formation of $\mathrm{Ti}$ 

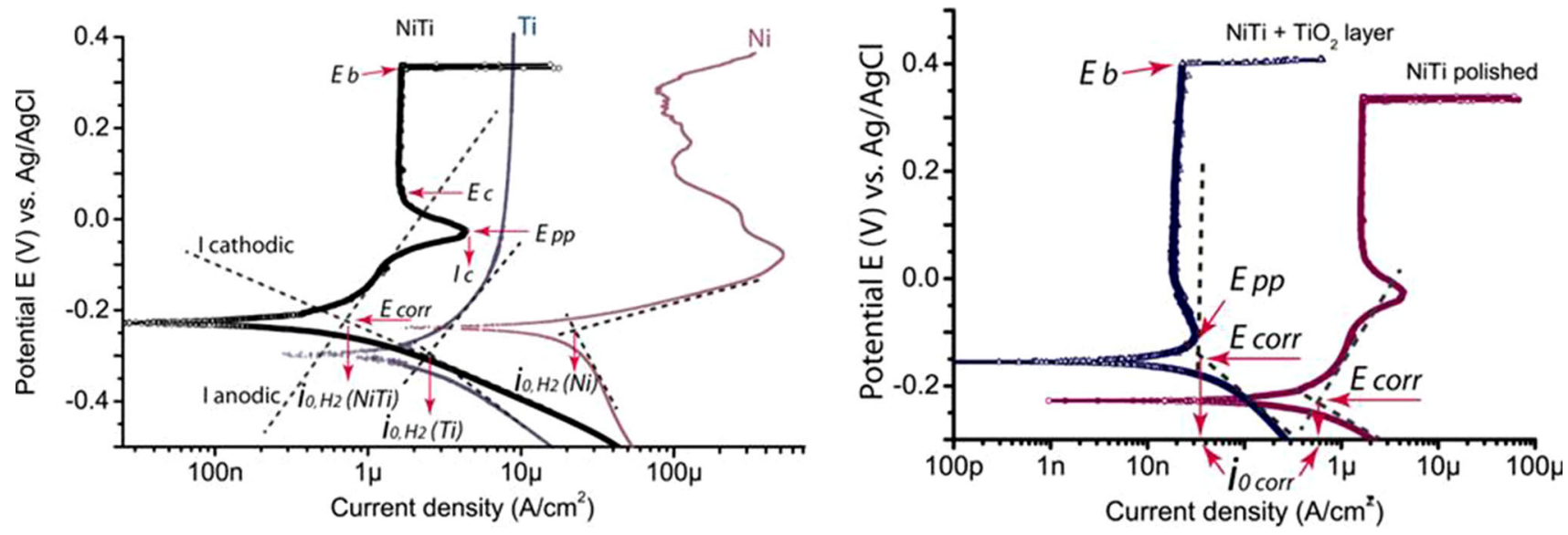

Fig. 21 Potentiodynamic polarization curves of a $\mathrm{NiTi}\left(\mathrm{TiO}_{2}\right.$ oxide layer $\sim 10 \mathrm{~nm}$ ), nickel and titanium wires, b heat-treated NiTi wire $\left(\mathrm{TiO}_{2}\right.$ oxide layer $\left.\sim 70 \mathrm{~nm}\right)$ used in present experiments and a mechanically polished NiTi wire $\left(\mathrm{TiO}_{2}\right.$ oxide layer $\left.\sim 10 \mathrm{~nm}\right) . E_{\mathrm{b}}$ denotes breakdown potential. $E_{\text {corr }}$ corrosion potential, $E_{\mathrm{pp}}$
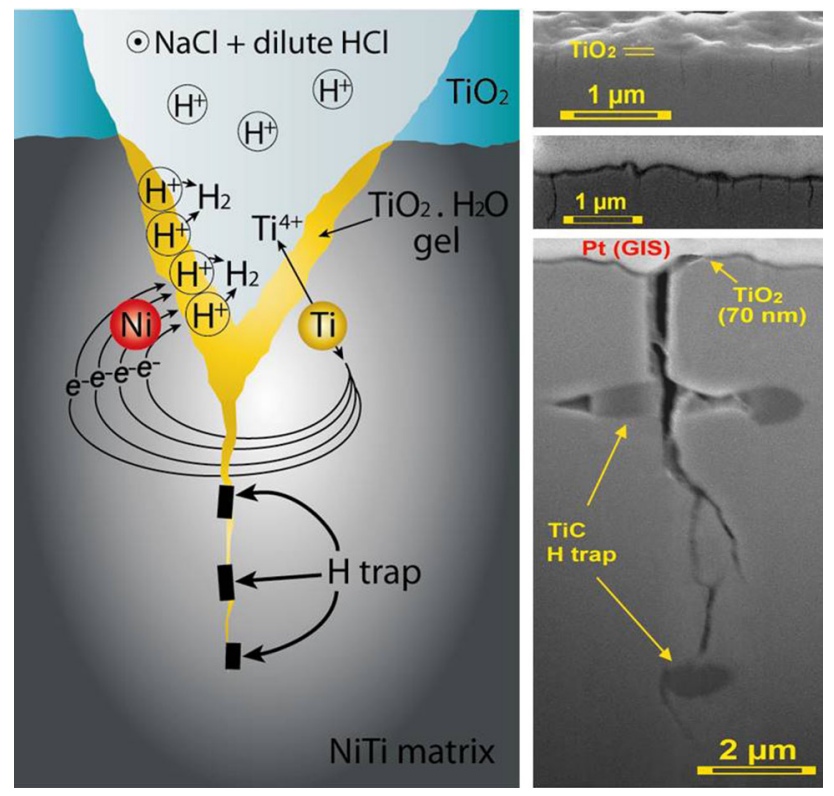

Fig. 22 Schematic drawing describing mechanically driven chemical reactions at the tip of the crack propagating into the NiTi matrix underneath the $\mathrm{TiO}_{2}$ oxide cracks opening/closing during the cyclic tensile deformation. The $\mathrm{TiO}_{2} \cdot \mathrm{H}_{2} \mathrm{O}$ gel created by the reactions at the newly open bare NiTi surface is suggested by yellow color. The hydrogen created by the reactions becomes adsorbed by the NiTi matrix, transported into NiTi bulk (brighter area) via diffusion enhanced by the interfaces and dislocations moving during the phase transformation. The hydrogen locally suppresses martensitic transformation temperature, concentrates in traps, and possibly forms titanium hydride in affected areas. All oxide cracks gradually grow into the NiTi matrix upon cycling (upper right figure). Major matrix cracks cut through or grow around the inclusions (same wire as in Fig. 20) (Color figure online) passivation potential, $E_{\mathrm{c}}$ critical potential, $I_{\mathrm{c}}$ critical current. The dashed lines are slopes of potentiodynamic polarization curves approaching the minimum electric current. They meet at the open circuit potential characterizing the surface quality of the virgin NiTi wire

hydrides [35] would lead to the acceleration of the crack growth and promotion of brittle fracture of superelastically cycled NiTi. However, this is the case for the intentionally hydrogenated NiTi. The hydrogen-free NiTi immersed in biofluids should be perfectly safe from the hydrogen embrittlement, as generally argued in the literature [18-20, 42].

At the same time, when the NiTi wire is exposed to the biofluid for a long time, as is the case of medical implants, it is possible that the corrosive chemical processes proceeding for a long time may decrease the strength of the NiTi wire. Inspection of the fractured wire segments from explanted esophageal NiTi stents reported in the literature $[16,17]$ confirm significant corrosion damage. This apparently supported the widespread view within the community that corrosion has to be taken seriously as the possible origin of the apparently random NiTi fractures. We have performed a series of long-time immersion tests, in which this NiTi wire was exposed to various simulated biofluids in virgin state as well as in deformed state (wire was wound around $2 \mathrm{~mm}$ plastic bar) for up to 72 days. No effect of the long-time immersion on strength and functional properties of the NiTi wire was found at all. This excluded possible effect of the chemical attack during the long-term corrosion on the strength even for immersed NiTi wire, including the wire with cracked surface oxide and stress-induced martensite in the subsurface matrix.

As introduced in the section "Mechanochemical reactions at the NiTi surface," hydrogen is produced by the mechanically driven chemical reactions $(5,6)$ triggered by 


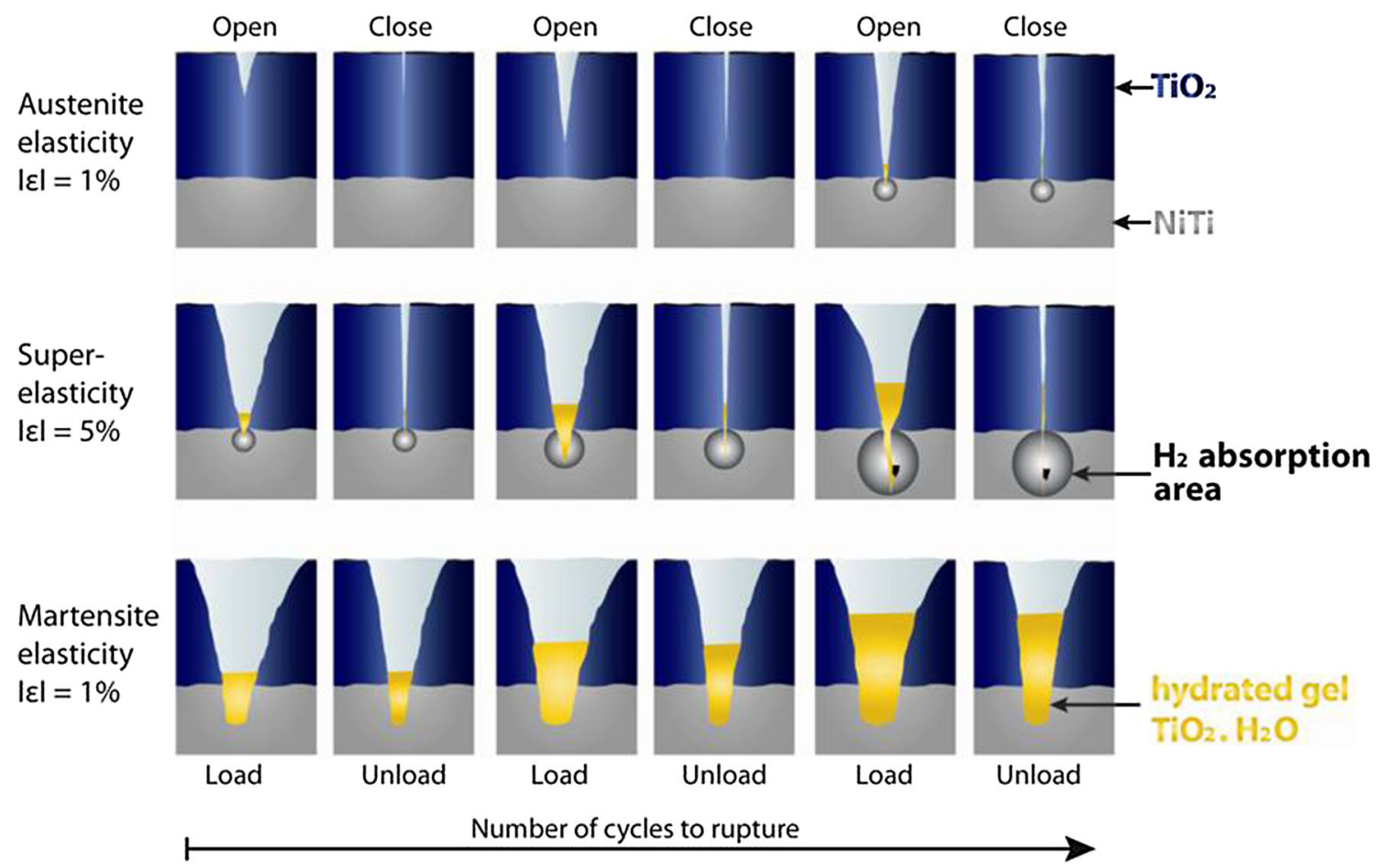

Fig. 23 Schematic figure suggests accumulation of the fatigue damage during cyclic tensile loading of NiTi wire by applying small strain amplitude in tests carried out using three different mean strains $0.5 \%$ (top), $4 \%$ (middle), and $7.5 \%$ (bottom) (e.g., in Fig. 14)

the electrolyte coming into contact with the bare NiTi within the oxide cracks as suggested in Fig. 22. The oxide/ NiTi matrix interface was investigated by atomistic modeling in Ref. [43]. An interesting structural relaxation giving rise to a columnar structure in which alternating long and short $\mathrm{Ni}-\mathrm{Ti}$ bonds are present in each surface and subsurface plane is reported. A thermodynamic analysis suggested that the most stable interface structure is that with Ti vacancies in the NiTi surface. This is in perfect agreement with frequent literature evidence [14, 18] for $\mathrm{Ni}$ rich layer in the subsurface NiTi matrix.

Such oxide/alloy interface structure would be relatively open for hydrogen adsorption. The solubility of hydrogen in liquids is greater at low temperatures [44]. Both adsorption and dissociative chemisorption of hydrogen accompanying the crack growth suggested in Fig. 22 depend on hydrogen charging conditions, temperature, and mechanical loading, which cannot be understood without performing dedicated experiments.

The mechanically driven chemical reactions $(5,6)$, however, can only lead to the hydrogen embrittlement, if sufficient amount of hydrogen is generated at the surface and if this hydrogen diffuses into the bulk at the $37{ }^{\circ} \mathrm{C}$ temperature. Due to the strong potential dependence, extreme hydrogen concentrations can be accommodated just underneath the surface [45]. Concerning the hydrogen diffusion in the NiTi matrix, martensitic phase transformation makes the assessment complicated. It is well known that atomic hydrogen in metals tends to be concentrated in the so-called "hydrogen traps." Most of the structural inhomogeneities, as grain boundaries or dislocations, act as hydrogen traps. The hydrogen trapping might be reversible or irreversible, depending on whether the trapped hydrogen is easily released or tightly bounded. From this point of view, nanosized partially recrystallized microstructure of heavily coldworked medical grade NiTi wires [9] with its grain boundaries, defects, and internal stresses should absorb hydrogen more easily than the fully annealed NiTi. Martensite with internal twins and variant interfaces should be theoretically even more open for hydrogen diffusion than the austenite phase.

Additionally, the phase-transforming NiTi contains high density of moving phase interfaces that generate slip dislocations during cyclic mechanical loadings $[9,10]$. Assuming that the hydrogen diffuses along the core of the dislocations within the moving interfaces [32], one can imagine a very efficient hydrogen transport from the surface to the bulk along the paths, where the interfaces repeatedly move during the cyclic superelastic deformation. Such mechanism would explain the experimental results of Yokoyama et al. [39, 40]. Considering that the adsorbed hydrogen locally suppresses the martensitic transformation $[35,41,46]$, one can deduce that the local internal stress necessary to drive martensitic transformation 
in the hydrogenated areas gradually increases with the increasing hydrogen content. Eventually, the crystal lattice might locally stop to deform superelastically, which would accelerate propagation of cracks in the hydrogenated areas (Fig. 22). Similarly hydrides and hydrogen traps would benefit crack growth (Fig. 22 right).

Since the hydrogen is produced only during the short time after the oxide damage event when the electrolyte reaches the bare NiTi surface, it is evident that the surface oxide damage must occur repeatedly in a cyclic manner in order to assure sufficient hydrogen production. At the same time, very efficient hydrogen transport to the bulk will be assured by the periodically moving phase interfaces and dislocations, as introduced above. Hydrogen transport by periodically moving interfaces and dislocations leads to enhanced hydrogen concentrations at inhomogeneities as grain boundaries or inclusions, where hydrogen traps form. Both conditions are ideally fulfilled in parts of the wire, where shear band fronts periodically move upon tensile cycling.

The periodical oxide damage is localized at the crack tips (Figs. 1, 22, 23), while the uncracked oxide areas among the cracks probably passivate continuously upon cycling, equally as the areas unreached by the moving shear band fronts. Because of this, the opening/closing oxide cracks under the moving shear band fronts are assumed to be the locations of preferential hydrogen production, the matrix cracks are assumed to be initiated there and this is where the wire ultimately fractures, when major cracks develop and grow deep into the matrix.

\section{Mechanochemical Reactions upon Cycling Evidenced by the In Situ OCP}

As the shear band front(s) move forward and back during the cyclic superelastic tensile loading, the surface oxide cracks open/close in a response to the surface strain varying from 1 to $7 \%$. While the strain increases, fresh new bare NiTi surface becomes periodically exposed to the electrolyte (Figs. 1, 20, 22, 23). The experimentally measured OCP provides information about the chemical processes triggered by the mechanical damage at the surface. Although the OCP starts to decrease already in the elastic range before the shear band is nucleated (Figs. 4, 5, 6, 7, 8), major drop of the OCP is related to shear band nucleation and consequent oxide cracking. The NiTi surface, however, has intrinsic ability to recover the mechanically induced damage by the passivation (chemical reaction 5) pushing the OCP to higher values and restoring thus the electrochemical equilibrium at the solid/ liquid interface. This scenario was deduced from the analysis of the results of the kinetics tests (Figs. 4, 5, 6, 7, 8). When the wire was deformed till elastic strains only, the surface oxide was deformed elastically but not broken. OCP decreased in response to the cyclic straining. This probably introduced into the oxide layer a homogeneously distributed damage but not the cracks. There was no sharp OCP minimum since there was no subsequent passivation after the damage (see kinetics tests in Figs. 6a, 7a). The existence of the OCP minimum on OCP-time curves recorded in kinetics tests up to larger applied strains is thus considered to be related to the massive oxide cracking accompanying the shear band nucleation. Once the surface oxide fractures and the bare NiTi surface is suddenly exposed to the electrolyte, OCP sharply fells but, at the same time, the passivation reaction starts pushing the OCP up again. The increase of the OCP [lasts only for $\sim 250 \mathrm{~ms}$ in the fast kinetics tests (Fig. 7)] thus evidences the massive oxidation taking place at the bare metal surface at the opened crack tips. When the tensile deformation is imposed slowly (Fig. 6) in the kinetics test, the situation is more complex due to the overlapping kinetics of the reactions proceeding in various locations on the nonhomogeneously deforming wire surface. The oxidation reaction occurs already, while the wire is being deformed and short time after that ( $\sim 250 \mathrm{~ms})$ (Fig. 6c, d). Recall that the oxidation reaction is responsible for the hydrogen generation. The mechanically driven chemical reactions thus effectively work as a kind of "pump supplying atomic hydrogen to NiTi," as introduced in "Mechanochemical reactions at the NiTi surface" section.

Since the oxidation processes are inhomogeneously distributed over the surface of the inhomogeneously deforming wire, the OCP kinetics curves (Figs. 4, 5, 6, 7) can only be interpreted via dedicated modeling based on the kinetics Eq. 4. The overlapping damage and passivation during the kinetics tests also rationalizes the experimentally observed dependence of the value of the OCP minimum on the imposed strain and strain rate (Fig. 8). The results of the modeling of the kinetics tests are going to be reported in a separate article.

Selected results concerning cyclic elastic loading of the NiTi wire in austenite state, where the surface damage is supposed to be homogeneous, are shown in Fig. 9a, b. Based on the gradual decrease of the OCP in the first 30 cycles (Fig. 9b), it can be deduced that the damage is introduced into the oxide layer gradually (Fig. 23 top). The OCP starts to increase when the passivation reaction prevails over the damage. The NiTi wire exhibits much stronger tendency for the passivation than the Tungsten wire. Although this is very interesting, we will not pay further attention to the cyclic loading of NiTi in the elastic range, since this article focusses on the superelastic fatigue.

As introduced above, the essential problem for the analysis of the OCP recorded during the superelastic cycling is that the experimentally recorded evolution of the OCP reflects the progress of mechanochemical reactions integrated over the whole surface of the wire but the shear band nucleation and propagation during the superelastic 
deformation are strongly localized in space and the shear band front(s) move. The evolution of the OCP recorded during the superelastic test (Figs. 10,11) is thus very different from that observed in the elastic loading test where the deformation is homogeneous. The OCP fells to a deep minimum during the first forward tensile loading and then gradually increases upon further cycling (Figs. 10c, 11a). Since the experimentally measured OCP is an integrated value over the whole wire surface, one cannot directly deduce what happens at the propagating shear band front (Fig. 1) from the experimentally recorded OCP-strain response during a single cycle (Fig. 11a). Dedicated modeling of the nonhomogeneous and time-dependent damage and passivation is again needed. In particular, even if we do not see any sharp OCP minimum on the OCPstrain curve, we anticipate that the oxide damage followed by passivation events (Eqs. 4, 5, 6) proceeds locally at the propagating shear band front and that this repeats during the tensile cycling. As the passivation reaction tends to dominate over the damage from the second cycle, the average OCP gradually increases upon cycling.

The surface of the NiTi wire subjected to superelastic cycling is thus exposed to the periodical sequence of the breakdown/passivation events. The essential point is that, within the periodically opening/closing oxide cracks (Fig. 23 middle), the bare NiTi matrix is repeatedly exposed to the biofluid which triggers the chemical reactions (Eqs. 5, 6) leading to hydrogen generation, as introduced in "Mechanochemical reactions at the NiTi surface" section. To confirm this role of the hydrogen, we applied negative electrochemical potential of $\sim-700 \mathrm{mV}$ during the superelastic fatigue test. This promoted the activity of the chemical reaction (5) which accelerated the hydrogen generation upon the tensile cycling. As a result, the number of cycles till failure was reduced to $\sim 600$.

\section{The Effect of Test Parameters on Fatigue Performance}

The results of the systematic investigation of the effect of various test parameters (strain rate (Figs. 12, 13), mean strain (Fig. 14), strain amplitude (Fig. 15), and temperature [Figs. 16, 17)] on fatigue performance show that there is a correlation between the in situ evaluated OCP and number of cycles till failure. Some of the results as discussed below are intriguing.

Very surprising is the fatigue performance of the wire improving with the increasing mean strain (Fig. 14). The number of cycles till failure in case of the $7.5 \%$ mean strain is about 15 times larger than for the mean strain $4 \%$ and this is very reproducible. How can it be? Recall that in case of $0.5 \%$ mean strain and $7.5 \%$ mean strain, there are no shear bands and the deformation is homogeneous. In case of the $0.5 \%$ mean strain, the wire is cycled in austenite elastic range and the OCP decreases gradually upon cycling (Fig. 14c). The damage is thus expected to prevail over passivation till $\sim 30$ th cycle (Fig. 23 top), when the passivation starts to prevail and OCP starts to increase. In case of the mean strain $4 \%$, the situation is very different. The shear band front(s) somewhere in the wire (Fig. 1) move very little in response to the externally imposed small strain amplitude, as can be deduced from the marginal stress hysteresis observed in Fig. 14b. Although the damage is localized into the very narrow region, where the shear band front(s) move, it results in lower OCP recorded in the 100th cycle (Fig. 14b). The highest OCP value observed in case of the mean strain $7.5 \%$ can be rationalized by assuming that passivation reaction in the widely opened crack (Fig. 23 bottom) leads to accumulation of the $\mathrm{TiO}_{2}$ gel at the crack tip and steady increase of the OCP upon cycling. The bare NiTi surface is not directly exposed to the electrolyte in case of the mean strain $7.5 \%$, as a result of which the generation of hydrogen is minimal and fatigue performance dramatically improves in spite of largest strain and stress.

In addition, the above introduced "hydrogen pump" works homogeneously over the wire surface and the surface strain variations are rather small $(<1 \%)$ in case of the mean strain 0.5 and $7.5 \%$. But in the case of the $4 \%$ mean strain, hydrogen generation concentrates into the surface areas, where shear band front(s) move (Fig. 1) and the local surface strain variations are large $(\sim 6 \%)$. The localized hydrogen generation at the moving shear band front is much more effective since the strain is large. This is the key argument explaining the shortest lifetime observed in case of the $4 \%$ mean strain. Second argument, however, is the already mentioned fast hydrogen transport by the moving interfaces which is obviously active only in case of the $4 \%$ mean strain. Even if the "hydrogen pump" would produce same amount of hydrogen per cycle for all three mean strains, building upon this argument, the hydrogen would be fastest adsorbed and transported into the bulk in case of the $4 \%$ mean strain. Due to the hydrogen-induced loss of the strength, the number of cycles till failure would be lowest in this case. Third argument for the shortest lifetime of the wire cycled at the $4 \%$ mean strain is based on the concentration of stress in location where the shear band front intersects with the wire surface. We have recently evaluated the topology of the shear band front and stresses around it experimentally by performing a unique 3D XRD experiment on thin NiTi wire stretched in tension [47]. It shall be mentioned that the increasing number of cycles till failure with the increasing mean strain was already reported in the literature before, e.g., by Pelton et al. [12], who performed fatigue tests on stent-like V-shape specimens processed from Nitinol microtubing. Since there are no shear bands in case of the 
V-shape samples, the observation of better fatigue performance of NiTi at larger mean strains in the martensite state in yet some other studies [2] might be of general relevance.

The increase of number of cycles till failure with the increasing strain amplitude (Fig. 15) is even more curious. Although this is ridiculous from the point of view of conventional fatigue behaviors of metals, it is perfectly understandable in the context of the hydrogen embrittlement due to mechanically driven chemical reactions on the surface of $\mathrm{NiTi}$ wire deforming via shear band front propagation. To break the wire, there is no need to make it brittle everywhere along its length, it is enough if this occurs in places where the shear band front moves. The larger is the strain amplitude, the less negative is the OCP (Fig. 15b), meaning that the passivation is more effective. As the strain amplitude gets smaller, the "hydrogen pump" operates efficiently only in the part of the surface swept periodically by the moving shear band front(s). Since the OCP continuously increases in the rest of the wire surface, the integral OCP eventually becomes least negative in case of the smallest strain amplitude. Locally, however, in the part of the wire regularly swept by the moving shear band front(s), the mechanochemical reaction might work even more efficiently at smaller strain amplitudes, since there is little time left for the passivation. The second argument (the hydrogen uptake by phase-transforming wire) and third argument (the increased stress at the shear band front) proposed in the previous paragraph would favor the increasing fatigue lifetime with the increasing strain amplitude as well. It comes out, that to assess the fatigue performance of superelastic NiTi wire in cyclic tension, it is very useful to plot fatigue limit in the mean strain-strain amplitude space, as proposed by Pelton et al. [12]. We did not do it since we did not have enough data points.

The number of cycles till failure decreasing with the increasing strain rate (Figs. 12,13) is not common as well. The effect is, however, relatively small. The decrease of the OCP with the increasing strain rate (Figs. 12a, 13) is evident. Since the passivation is a time-dependent process, there is simply less time for it in tests performed with higher strain rates. Whether this is directly related to the decreasing number of cycles till failure or not is, however, questionable. The strain rate also affects the superelastic response (Fig. 13) and its evolution upon cycling (Fig. 12d). The higher transformation stress at higher strain rates most likely originates from the impact of the latent heat effects on superelasticity [11] and fatigue performance might worsen with the increasing stress (Fig. 12d).

Finally, the increasing temperature has negligible effect on the in situ recorded OCP (Figs. 16, 17) suggesting that the effect of temperature on the mechanochemical reactions at the surface is marginal. The number of cycles till failure, nevertheless, decreases with the increasing temperature. The decrease of the fatigue lifetime with the increasing temperature might be due to the transformation stress increasing with the increasing temperature.

\section{Damage Accumulation and Fracture}

What is it actually the "damage" evidenced by the sharply decreasing OCP? Neglecting the early elastic range, where it could be related to the stresses in the surface oxide, the damage consists mainly in the oxide cracking. Later, when cracks appear at the surface and grow into the NiTi matrix, the oxide gel at the crack tip is disrupted by the straining and exposes the bare metal to the liquid again and again (Figs. 22, 23). In this respect, major damage occurs in the first superelastic cycle when the virgin oxide is firstly broken into the cracked oxide layer with millions of nanometer-sized cracks (Fig. 1).

The oxide cracks periodically open/close upon tensile cycling (Figs. 19, 20) and the damage/passivation events (Figs. 22, 23) are localized there. The SEM observation of the small cracks on the surface showing one-to-one correspondence with the small cracks observed on cross section of the fatigued wire (Fig. 22) clearly supports that view. The fact that the OCP increases during superelastic tests suggests that the surface damage is in fact gradually "cured" in spite of the cyclic mechanical loading. The real irreversible damage, however, cannot be detected by the surface SEM observations, since it consists in the local hydrogen embrittlement of the NiTi matrix underneath the oxide cracks enabled by the mechanochemical reactions, nucleation of matrix cracks, and their growth in the embrittled NiTi lattice.

In addition, there are those major cracks appearing frequently at inclusions and notches on the surface of the fatigued wire (Fig. 19), which penetrate several microns deep into the NiTi matrix (Fig. 20). This can be readily understood based on the fact that the hard elastic inclusions that cannot deform together with the superelastic NiTi matrix act as natural stress concentrators. On top of that, it is likely that the hydrogen adsorption can be accelerated at the oxide or carbide inclusions on the surface (Fig. 19) and that the inclusions thus act as a kind of "surface hydrogen traps." This would explain why major cracks, the propagation of which leads to the fracture, are frequently connected to the surface inclusions in view of the hereabout introduced mechanism.

In summary, the proposed mechanism for fatigue failure of superelastically cycled NiTi wires in simulated biofluids involves the following 8 steps:

1) fracture of the $\mathrm{TiO}_{2}$ surface layer by dense network of thin oxide cracks,

2) periodical opening/closing of the oxide cracks exposing the bare NiTi repeatedly to the electrolyte,

3) hydrogen generation by the mechanochemical reaction (passivation) at the NiTi/liquid interface, 
4) uptake of the atomic hydrogen by the bare NiTi surface,

5) transport of the hydrogen into bulk NiTi enhanced by the periodically moving phase interfaces during the cyclic martensitic transformation,

6) local loss of the strength of the NiTi matrix due to the hydrogen embrittlement and suppression of martensitic transformation,

7) transfer of the oxide cracks into the embrittled NiTi matrix,

8) propagation of selected cracks along the hydrogen traps causing ultimately the brittle fracture of the wire.

Ironically, combination of the two exciting properties of NiTi-superelasticity due to martensitic transformation and biocompatibility due to $\mathrm{TiO}_{2}$ surface oxide layer-thus leads to accelerated fatigue damage during cyclic loading in biofluids. The hydrogen-based fatigue degradation mechanism is proposed especially for NiTi, other SMAs, as e.g., $\mathrm{Ti}-\mathrm{Nb}-\mathrm{Ta}$ or $\mathrm{Ti}-\mathrm{Nb}-\mathrm{Zr}$ medical alloys might behave differently as recent electrochemical studies [48] show. A question remains whether the mechanism may also operate in atmospheric moisture.

The essential point is that the mechanism originates from the surface not from the microstructure evolution in the bulk, though the bulk deformation processes play significant role in it, since they allow for the excessive surface deformation and for the hydrogen transport. The mechanism might potentially pose a threat for any low modulus Ti alloy-based implants covered by $\mathrm{Ti}$ oxides. However, note that medical implants designed as a "structural elements" undergo much smaller surface strains than the thin NiTi wire and that the cyclic martensitic transformation required in step $\mathrm{v}$ is missing. Another likely consequence of the mechanochemical reactions during the superelastic cycling of NiTi wires immersed in biofluids is presumably a drastic increase of the Ni release from the cycled wire compared to the static NiTi wire (virgin or deformed [23] ). This shall be taken as a prediction that should be verified by dedicated experiments.

It shall be mentioned that the local hydrogenation and consequent decrease of the strength of NiTi due to the cyclic superelastic deformation in fluids is not the same as the homogeneous hydrogenation of NiTi by conventional means [38-42, 46, 49, 50]. Minor homogeneous absorption of hydrogen by NiTi does not have to necessarily decrease its strength. It may have even beneficial effect on, e.g., corrosion [38] or damping properties [49]. Hydrogenated NiTi recovers its strength upon long-time aging even at room temperature [50].

Finally, it shall be pointed out that fatigue performance of the thin NiTi wires in cyclic tensile tests depends critically on the type and quality of the oxide layer resulting from the surface finishing treatment. NiTi wires with different oxide layers exhibit very different number of cycles till failure. Recall that all samples studied in this work had exactly the same surface oxide layer achieved by the same heat treatment. We shall admit that this particular oxide layer [14] definitely did not provide the wire with the best fatigue performance. But this is not considered to be an issue here, since the goal of this work was to reveal the degradation mechanism and to develop a method that can be used to evaluate various surface finishing treatments of NiTi wires in view of the best superelastic fatigue performance in simulated body fluids. Fatigue testing of NiTi wires having same microstructure but various surface oxides due to various surface finishing treatments is currently in progress.

\section{Conclusions}

Fatigue of oxidized superelastic NiTi wires was investigated by cyclic tensile tests in simulated biofluid. Although the static NiTi wire is highly corrosion resistant thanks to the thin passive $\mathrm{TiO}_{2}$ oxide layer covering its surface, the same NiTi wire, when strained cyclically in the liquid, suffers from environmentally induced fatigue degradation and preliminary failure. As the thin $\mathrm{TiO}_{2}$ surface oxide cannot withstand the large superelastic strains, the wire is in fact covered by a "cracked surface oxide" containing dense array of regularly spaced microcracks that open/close periodically upon cycling and expose fresh NiTi surface to the liquid. Fatigue lifetime of superelastically NiTi wire is shorter in the liquid compared to the air.

The state of the surface of the cyclically deformed NiTi wire was continuously monitored by following the evolution of the electrochemical Open Circuit Potential alongside with the macroscopic stress and strain. The OCP signal reflects mechanically induced local breakdown of the passive oxide layers triggering the chemical reactions on the wire surface. Microcracks on the surface of the stretched fatigued wire as well as on the FIBed cross section were observed by SEM. Based on the results, it is claimed that fatigue lifetime of superelastic NiTi wire deforming cyclically in simulated biofluid is controlled by mechanically driven chemical reactions (passivation) at the excessively stretched surface leading to hydrogen generation at the tips of the periodically opening/closing cracks (freshly opened bare NiTi/liquid interface).

Mechanism of environmental fatigue degradation of NiTi is proposed consisting in the adsorption of the generated hydrogen by the mechanically loaded surface, diffusional transfer of the adsorbed hydrogen into the bulk facilitated by the interfaces moving during the cyclic martensitic transformation, and local suppression of the 
martensitic transformation in hydrogen-affected areas. This mechanism assists crack nucleation under the surface oxide cracks and facilitates growth of selected cracks into the matrix, which ultimately leads to the brittle fracture of the NiTi wire. Major cracks always nucleate at inclusions or notches on the surface.

The mechanism explains the origin of clinical observations of random embrittlement and fractures of NiTi wires taken from explanted tracheal and esophageal NiTi stents.

Acknowledgments Support from the research Projects GAČR 14-36566G (J. Racek), P107/12/0800 (L. Heller), and GA14-15264S (P. Sittner) of the Grant Agency of the Czech Republic and project FUNBIO - CZ.2.16/3.1.00/21568 is acknowledged. The authors thank Ing. J. Pilch and Ing. L. Kaderavek for their assistance with the design and fabrication of the in situ electrochemical loading rig and Ing. K. Hiřmanová, who carried out the long-time immersion tests. The authors thank the company TESCAN ORSAY HOLDING for assistance with special SEM experiments.

\section{References}

1. Jani JM, Leary L, Subic A, Gibson MA (2014) A review of shape memory alloy research, applications and opportunities. Mater Des 56:1078-1113

2. Figueiredo AM, Modenesi P, Buono V (2009) Low-cycle fatigue life of superelastic NiTi wires. Int J Fatigue 31:751-758

3. Eggeler G, Hornbogen E, Yawny A, Heckmann A, Wagner M (2004) Structural and functional fatigue of NiTi shape memory alloys. Mater Sci Eng, A 378:24-33

4. Maletta C, Sgambitterra E, Furgiuele F, Casati R, Tuissi A (2014) Fatigue properties of a pseudoelastic NiTi alloy: strain ratcheting and hysteresis under cyclic tensile loading. Int J Fatigue 66:78-85

5. Tabanli RM, Simha NK, Berg BT (2001) Mean strain effects on the fatigue properties of superelastic NiTi. Metall Mater Trans A 32:1866-1869

6. Robertson SW, Pelton AR, Ritchie RO (2012) Mechanical fatigue and fracture of Nitinol. Int Mater Rev 57:1-37

7. Chao Yu, Kang Guozheng, Kan Qianhua (2014) A physical mechanism based constitutive model for temperature-dependent transformation ratcheting of NiTi shape memory alloy: one-dimensional model. Mech Mater 78:1-10

8. Moumni Z, Van Herpen A, Riberty P (2005) Fatigue analysis of shape memory alloys: energy approach. Smart Mater Struct 14:S287-S292

9. Norfleet DM, Sarosi PM, Manchiraju S, Wagner MF-X, Uchic MD, Anderson PM, Mills MJ (2009) Study of transformation induced plasticity during pseudo elastic deformation of $\mathrm{NiTi}$ microcrystal. Acta Mater 57:3549-3561

10. Delville R, Malard B, Pilch J, Sittner P, Schryvers D (2011) Transmission electron microscopy investigation of dislocation slip during superelastic cycling of $\mathrm{Ni}-\mathrm{Ti}$ wires. Int $\mathrm{J}$ Plast 27:282-297

11. Heller L, Sittner P, Pilch J (2013) Impact of heat effects on superelasticity, In: Olson GB, Lieberman DS, Saxena A (eds) International Conference on Martensitic Transformations (ICOMAT)), Wiley, Hoboken, 2010, Santa FE, USA, Wiley. doi:10. 1002/9781118803592.ch64

12. Pelton AR, Schroeder V, Mitchel MR, Gong XY, Barney M, Robertson SW (2008) Fatigue and durability of nitinol stents. J Mech Behav 1:159-164
13. Hiřmanová K, Pilch J, Racek J, Heller L, Šittner P, Sedlák $P$ (2014) Physical simulation of the random failure of implanted braided NiTi stents. J Mater Eng Perform 23:2650-2658

14. Racek J, Šittner P, Heller L, Pilch J, Petrenec M, Sedlák P (2014) Corrosion of NiTi wires withy cracked oxide layer. J Mater Eng Perform 7:2659-2668

15. Sedlák P, Frost M, Hiřmanová K, Šittner P, Heller L (2014) Simulations of mechanical response of superelastic NiTi helical spring and its relation to fatigue resistance. J Mater Eng Perform 7:2591-2598

16. Wiedmann M, Heller F, Zeitz M, Mössner J (2009) Fracture of a covered self-expanding antireflux stent in two patients with distal esophageal carcinoma. Endoscopy 41:E129-E130

17. Rana SS, Bhasin DK, Sidhu GS, Rawal P, Nagi B, Singh K (2009) Nitinol stent dysfunction related to fracture and collapse. Endoscopy 41:E170-E171

18. Shabalovskaya S, Anderegg J, Humbeeck JV (2008) Critical overview of Nitinol surfaces and their modifications for medical applications. Acta Biomater 4:447-467

19. Pound BG (2014) Corrosion behavior of metallic materials in biomedical applications. I. Ti and its alloys. Corros Rev 32:1-20

20. Chen Q, Thouas GA (2015) Metallic implant biomaterials. Mater Sci Eng R 87:1-57

21. Firstov GS, Vitchev RG, Kumar H, Blanpain B, Humbeeck JV (2002) Surface oxidation of NiTi shape memory alloy. Biomaterials 23:4863-4871

22. Diebold U (2003) Surface science of titanium dioxide. Surf Sci Rep 48:53-229

23. Freiberg KE, Bremer-Streck S, Kiehntopf M, Rettenmayr M, Undisz A (2014) Acta Biomater 5:2290-2295

24. Tanner RE, Goldfarb I, Castell MR, Briggs GAD (2001) The evolution of $\mathrm{Ni}$ nanoislands on the rutile $\mathrm{TiO} 2(110)$ surface with coverage, heating and oxygen treatment. Surf Sci 486:167-184

25. Figueira N, Silva TM, Carmezn MJ, Fernandez JCS (2009) Corrosion behaviour of NiTi alloy. Electrochim Acta 54:921-926

26. Xu LY, Cheng YF (2012) Corrosion of X100 pipeline steel under plastic strain in a neutral $\mathrm{pH}$ bicarbonate solution. Corros Sci 64:145-152

27. Neelakantan L, Zglinski JK, Frotscher M, Eggeler G (2013) Design and fabrication of a bending rotation fatigue test rig for in situ electrochemical analysis during fatigue testing of $\mathrm{NiTi}$ shape memory alloy wires. Rev Sci Instrum 84:035102

28. Perrin G, Dunaway W, Carter TS, McKennis J, Mishra SK, Morse AR Method for monitoring corrosion damage to a metal sample, US patent US0073 87031B1

29. Gutman EM (1994) Mechanochemistry of solid surfaces. World Scientific, Singapore

30. Hall MM Jr (2009) Film rupture model for aqueous stress corrosion cracking under constant and variable stress intensity factor. Corros Sci 51:225-233

31. Racek J, Šittner P, Heller L, Pilch J, Kadeřávek L (2015) Electrochemistry of NiTi wires/springs subjected to static/cyclic loadings. In: Proceedings of Icomat 2014, Materials Today Proceedings, in print

32. Shuh Ch (2000) Modelling gas diffusion into metals with a moving-boundary phase transition. Metall Mater Trans A 31A:2411-2421

33. Pourbaix M (1974) Atlas of electrochemical equilibria in aqueous solutions, NACE, 2nd English edn. Houston, Texas

34. Ezaki H, Morinaga M, Watanabe S (1993) Overpotential for transition metals and alloys, and its interpretation using an electronic model. Electrochem Acta 38:557-564

35. Soubeyroux JL, Fruchart D, Lorthioir G, Ochin P, Colin D (1993) Structural study of the hydrides NiTiHx ( $\mathrm{x}=1.0$ and 1.4). J Alloys Compd 196:127-132 
36. Emami H, Cuevas F (2011) Hydrogenation properties of shape memory $\mathrm{Ti}(\mathrm{Ni}, \mathrm{Pd})$ compounds. Intermetallics 19:876-886

37. Robertson M, Martin ML, Fenske JA (2012) Influence of hydrogen on the behavior of dislocations. In: Gangloff RP, Somerday BP (eds) Gaseous hydrogen embrittlement of materials in energy technologies, vol 2. Woodhead Publishing, Cambridge, pp 166-200

38. Yokoyama K, Hirata Y, Inaba T, Mutoh K, Sakai J (2015) Inhibition of localized corrosion of $\mathrm{Ni}-\mathrm{Ti}$ superelastic alloy in $\mathrm{NaCl}$ solution by hydrogen charging. J Alloys Compd 639: 365-372

39. Tomita M, Yokoyama K, Asaoka K, Sakai J (2008) Hydrogen thermal desorption behavior of $\mathrm{Ni}-\mathrm{Ti}$ superelastic alloy subjected to tensile deformation after hydrogen charging. Mater Sci Eng A 476:308-315

40. Yokoyama K, Tomita M, Sakai J (2009) Hydrogen embrittlement behavior induced by dynamic martensite transformation of $\mathrm{Ni}-\mathrm{Ti}$ superelastic alloy. Acta Mater 57:1875-1885

41. Runciman A, Chen CK, Pelton AR, Trepanier C (2006) Effects of hydrogen on the phases and transition temperatures of NiTi. In: Berg B, Mitchell MR, Proft J (eds) Proceedings of SMST 2006, May 7-11, 2006, Pacific Grove, California, USA, ASM International 2006, 185-196

42. Scheriff J, Pelton A, Pruitt LA (2005) Hydrogen effects in Nitinol fatigue. In: Helmus M, Medlin D (eds) Proceedings of ASM Materials \& Processes for Medical Devices, ASM International, pp 38-43

43. Nolan M, Tofail SAM (2010) The atomic level structure of the TiO2-NiTi interface. Phys Chem Chem Phys 12:9742-9750
44. Verhallen PTHM, Oomen LJP, Elsen AJJMVD, Kruger J (1984) The diffusion coefficients of helium, hydrogen, oxygen and nitrogen in water determined from the permeability of a stagnant liquid layer in the quasi-s. Chem Eng Sci 39:1535-1541

45. Vehoff H (1997) Hydrogen related materials problems. In: Wipf $\mathrm{H}$ (ed) Hydrogen in metals III: properties and applications, vol 48. Springer, Berlin, pp 215-274

46. Gamaoun F, Ltaief M, Bouraoui T, BenZineb T (2014) Effect of hydrogen on the tensile strength of aged Ni-Ti superelastic alloy. J Intell Mater Syst Struct 25:980-988

47. Sedmak P, Pilch J, Sittner P, Curfs C, Wright J (2015) Luders band front propagating in thin superelastic NiTi wire in tension characterized by in situ synchrotron 3D X-ray diffraction method. Int J Plast (in preparation)

48. Zhukova YuS, Pustov YuA, Konopatsky AS, Filonov MR, Prokoshkin SD (2014) Electrochemical behavior of novel superelastic biomedical alloys in simulated physiological media under cyclic load. J Mater Eng Perform 7:2677-2681

49. Biscarini A, Campanella R, Coluzzi B, Mazzolai G, Trotta L, Tuissi A (1999) Martensitic transitions and mechanical spectroscopy of Ni $50.8 \mathrm{Ti} 49.2$ alloy containing hydrogen. Acta Mater 47:4525-4533

50. Yokoyama K, Ogawa T, Takashima K, Asaoka K, Sakai J (2007) Hydrogen embrittlement of Ni-Ti superelastic alloy aged at room temperature after hydrogen charging. Mater Sci Eng A 466:106-113

51. Li Y, Laird C Methods and devices for electrochemically determining metal fatigue status. US patent, US005419201A 